Submitted to Ap.J.

\title{
A Model of Solar Flares and Their Homologous Behavior
}

\author{
G. S. Choe and C. Z. Cheng \\ Princeton Plasma Physics Laboratory, Princeton University, Princeton, NJ 08543-0451 \\ gchoe@pppl.gov,fcheng@pppl.gov
}

\begin{abstract}
A model describing physical processes of solar flares and their homologous behavior is presented based on resistive MHD simulations of magnetic arcade evolution subject to continuous shear-increasing footpoint motions. It is proposed in our model that the individual flaring process encompasses magnetic reconnection of arcade field lines, generation of magnetic islands in the magnetic arcade, and coalescence of magnetic islands. When a magnetic arcade is sheared, a current sheet is formed and magnetic reconnection can take place to form a magnetic island. A continuing increase of magnetic shear can trigger a new reconnection process and create another island in the underlying arcade below the magnetic island. The newborn island rises faster than the preceding island and merges with it to form one island. Before merging with the upper island is completed, the newborn island exhibits two different phases of rising motion: the first phase with a slower rising speed and the second phase with a faster rising speed. This is consistent with the Yohkoh observation by Ohyama \& Shibata (1998) of X-ray plasma ejecta motion. The first phase, in which reconnection of line-tied field in the underlying arcade is important, can be regarded to be related with the preflare phase. In the second phase, the island coalescence takes place, which creates an elongated current sheet below and enhances the reconnection rate of the line-tied arcade field. This phase is interpreted as the impulsive phase or the flash phase of flares. The obtained reconnection electric field is large enough to accelerate electrons to an energy level higher than $10 \mathrm{keV}$, which is necessary for observed X-ray emissions. After merging of the islands is completed, magnetic reconnection continues in the current sheet under the integrated island for rather a long period, which can be considered as the main phase of flares. The sequence of all these processes is repeated with some time interval while a shear-increasing motion continues. We propose that a series of these flaring processes constitutes a set of homologous flares. The time interval between successive flaring events depends on the energy input rate into the system, which is governed by the nature of the footpoint motion and the flux reconnecting rate. We have also investigated the destruction of a magnetic island in a system undergoing a decrease of magnetic shear. The result suggests that there is a critical value of magnetic shear for existence of a magnetic island in an arcade-like field configuration.
\end{abstract}


Subject headings: Sun: flares, MHD, methods: numerical

\section{INTRODUCTION}

Solar flares are intense, abrupt release of energy occurring usually in the vicinity of an active region where the magnetic field is stressed. A large flare can release over $10^{32} \mathrm{erg}$ of energy in an hour or so, which is regarded as part of the magnetic free energy. Based on the temporal evolution of the flare emission, the progress of a flare can be divided into several phases (e.g., Kane 1974; Priest 1982). In the preflare phase, which lasts about 10 minutes before the flare onset, enhanced thermal emissions from the coronal plasma are detected mostly in the soft X-ray (photon energy $<10 \mathrm{keV}$ or $0.1 \mathrm{~nm}<\lambda<2 \mathrm{~nm}$ ). During the flash phase, which lasts typically about 5 minutes, the intensity and area of the emission rapidly increase. Then, in the main phase, the intensity slowly declines over about an hour or sometimes as long as a day. Large flares also exhibit an impulsive phase before the main phase, lasting 10-100 seconds, during which hard X-ray $(\lambda<0.1 \mathrm{~nm})$ and microwave bursts are observed.

Flares can be morphologically classified into "two-ribbon flares" and "simple-loop flares." All major flare events are two-ribbon flares, which are on a much larger spatial scale than simple-loop flares and are often related with a prominence eruption and/or a coronal mass ejection (CME). They are characterized by two bright $\mathrm{H}_{\alpha}$ ribbons moving apart with hot X-ray plasma which is frequently seen as if joining the ribbons. Most small scale flares are simple-loop flares, in which a single magnetic loop brightens in X-rays and remains unchanged in shape and position throughout the flaring event. It was generally believed that different mechanisms are involved in the two types of flares. Two-ribbon flares were considered to occur by reconnection of open field lines above a magnetic arcade (Sturrock 1968) or by reconnection of stretched arcade field lines (Kopp \& Pneuman 1976). On the other hand, it was speculated that simple-loop flares are generated by merging of two flux ropes (e.g., Gold \& Hoyle 1960) or by various other mechanisms working in a single loop (e.g., Spicer 1977; Van Hoven 1981; Kan, Akasofu, \& Lee 1983). However, Masuda et al. (1994) found that the HXR (hard X-ray) source, which is regarded to be directly related with reconnection, is located above the SXR (soft X-ray) loop even in simple-loop flares as well as in large two-ribbon flares. This means that the general configuration of the flaring magnetic field is not different from what was supposed in the Kopp-Pneuman type model (Kopp \& Pneuman 1976). Later, Shibata et al. (1995) found that most flares observed by Yohkoh were associated with X-ray plasma ejecta (or plasmoid), some of which were launched well before the flare impulsive phase, and they inferred that the plasmoid ejection is not a consequence of the flare, but a cause of it. Based on these observations, Shibata (1998) proposed a plasmoid-induced-reconnection model, in which a fast reconnection responsible for a flare is triggered by the plasmoid ejection. However, the formation and acceleration mechanism of the plasmoid was left unaddressed in their model.

In the Kopp-Pneuman model (Kopp \& Pneuman 1976), the plasmoid, a magnetic island with helical field lines in 2D or a helical magnetic structure loosely connected to the solar surface in 
$3 \mathrm{D}$, is formed by reconnection of line-tied field lines in a magnetic arcade. This possibility has indeed been confirmed by several numerical simulations (Mikić, Barnes, \& Schnack 1988; Inhester, Birn, \& Hesse 1992; Mikić \& Linker 1994; Linker \& Mikić 1995; Choe \& Lee 1996b; Amari et al. 1996a). In this picture, the rise of the plasmoid can be considered as a process of approaching a new equilibrium after a change in field topology. Thus, the plasmoid must be eventually decelerated unless the reconnection of arcade field lines under the plasmoid is indefinitely continued. However, recent observations of plasmoid ejection do not support the idea that the plasmoid is entirely driven by magnetic reconnection underneath. Ohyama \& Shibata (1998) found from Yohkoh observations that an ejecta in an X-ray flare rises with a speed of $\sim 250 \mathrm{kms}^{-1}$ before the main peak of the hard X-ray emission and is accelerated during the impulsive phase to $\sim 500 \mathrm{kms}^{-1}$. Such a twostep acceleration process with a faster second phase cannot be interpreted within the scope of the conventional Kopp-Pneuman picture although the flare morphology based on observations supports the Kopp-Pneuman-like field configuration in the vicinity of and under the reconnection site. A possible way of resolving this seeming contradiction is proposed in this paper.

In this paper, we also provide an explanation on another interesting feature of flare phenomena, the recurrence of homologous flares. It is often observed that a series of solar flares take place repetitively in the same active region with essentially the same position and with a common pattern of development. Such flaring phenomena are called homologous flares. The time interval between successive flaring events varies from several hours to a few days (Stix 1989). Woodgate (1982) suggested that a majority of flares might be homologous in the sense that the footpoints reappear very near the same place. In the SMM Workshop (Kundu \& Woodgate 1986), it was even argued that flare homology must be included among the constraints on flare models (Hagyard et al. 1986). The homologous conditions common to several homologous flare sets are summarized by Woodgate et al. (1984) as follows:

(1) Preflare upflows in small loops near flare footpoints.

(2) Horizontal photospheric flows in the flare vicinity.

(3) High magnetic shear across polarity inversion lines near the footpoints.

(4) Magnetic disruption and mass outflow during the flare.

(5) Reformation of filaments (prominences) and the corona between flares.

Interestingly, these are also conditions of most flares whether they are homologous or not. Among these conditions, the magnetic shear (the third condition) has been considered as the most important condition for solar eruptive phenomena. The origin of magnetic shear, however, is still being debated. It could be shear-increasing footpoint motions or emergence of new flux of sheared magnetic field. The shear-increasing footpoint motions do not only imply motions parallel to the polarity inversion line, but also motions towards the polarity inversion line when a little magnetic shear is already present. The role of a prominence in flare occurrence (the fifth condition) still remains unclear. From observations of erupting prominences (e.g., Zirin 1988), we at least know 
that prominences contain highly wound helical field lines. Thus, it can be inferred that most of the magnetic helicity in the flare-bearing active region is concentrated in and around the prominence. The observation by Rust \& Kumar (1996) suggests that an increase of magnetic helicity of a prominence can lead to eruption of the prominence. Thus, the prominence reformation between flares (the fifth condition) is probably related with a change in magnetic helicity. Increase of magnetic helicity of a prominence can be achieved either by reconnection of sheared arcade field lines surrounding the prominence or by reconnection of prominence field lines with a flux rope of the same helicity emerging from below. Therefore, any shear-increasing process can also increase magnetic helicity of a prominence if magnetic reconnection is possible.

This paper presents a numerical MHD model of the evolution of a bipolar active region which undergoes flaring processes including homologous flares. We investigate the evolution of magnetic arcades in a resistive plasma subject to various shear-increasing footpoint motions. There have been a number of studies dealing with shearing footpoint motions in magnetic arcades (Mikić et al. 1988; Biskamp \& Welter 1989; Inhester et al. 1992; Mikić \& Linker 1994; Choe \& Lee 1996b; Amari et al. 1996a). However, all these studies have concentrated on the possibility of arcade reconnection and magnetic island formation. Studying the subsequent evolution of the arcade-island system is all the more important in understanding the mechanism of homologous flares. The subject of repetitive solar eruptions was studied by Linker \& Mikić (1995) by resistive MHD simulations. In their numerical simulation, a 2D magnetic arcade with an axisymmetric geometry is sheared with a partially open initial field configuration embedded in a steady outward plasma stream resembling the solar wind. The simulation reveals formation of a magnetic island by reconnection of arcade field lines, its expulsion into the solar wind and reconnection of open field lines. The series of these events are found to repeat with a time interval of about 400 hours, i.e., about 17 days. Moreover, no interaction between the magnetic island and its surroundings was found. Thus, this study may explain a long term evolution of an active region involving grand scale solar eruptions like coronal mass ejections (CMEs), but does not pertain to homologous flares.

In our study, we assume a closed initial field configuration and focus on the evolution of the magnetic island not totally expelled from the sun. Because prominences are believed to reside at the bottom of a magnetic island (Kuperus \& Raadu 1974; Anzer 1979), a newborn island can be considered as a prominence newly appearing in the solar atmosphere. When a magnetic island is formed by reconnection of an arcade field, the toroidal flux originally contained in line-tied flux tubes is redistributed into two flux systems: the magnetic island and the underlying arcade with the reconnected line-tied field. The magnetic shear is thus reduced in the underlying arcade after island formation. A further shearing motion increases the magnetic shear in the lower flux system, and above a critical value of shear magnetic reconnection takes place to form a new magnetic island. The newborn magnetic island then rises and merges with the overlying magnetic island to form a single integrated island. During this process, the newborn magnetic island exhibits two phases of rising motion with different speeds (a slower initial rising phase followed by a faster rising phase) corresponding to the observed two phases of the plasmoid motion reported by Ohyama 
\& Shibata (1998). The reconnection electric field in the current sheet under the magnetic island system increases with the rising of the newborn island, reaches a maximum on the completion of island merging and gradually declines for a rather long duration. Thus, the phase of the new island creation and its slow rising is regarded as the preflare phase, the fast island rising phase involving the island coalescence is interpreted as the impulsive (or flash) phase, and the phase with a longer period of reconnection under the integrated island is considered as the main phase of a flare. The sequence of the above reconnection processes can be repeated as long as the magnetic shear is replenished, and we propose that a series of this reconnection sequence is a set of homologous flares. Also, we investigate the cause-effect relationship among those different reconnection processes to explain the mechanism of solar flares and their homologous nature.

It is important to comment on why we model the generation of magnetic shear by footpoint motions in this paper. This is not because we ignore the possibility of emergence of sheared flux tubes, but because we want to investigate the toroidal flux variation first. The complexity due to the change in poloidal flux resulting from flux emergence will be addressed in the future study. It should be emphasized that as long as the preflare evolution is quasi-static and undergoes little change of field topology until the flare onset, the models based on one process of shear buildup are believed to provide quite general information about the critical condition for flare development, which may be applicable to flares originating in other types of shear-increasing processes.

A sheared magnetic state can be approached either by a shearing motion parallel to the polarity inversion line or by a converging motion exerting on a pre-sheared field. Of course, there are indefinite number of possible paths approaching a given sheared configuration. In the photospheric observations during a preflare stage or during prominence formation, shearing mass motions are not so evidently detected as the magnetic shear is (Livi et al. 1989; Martin 1990). Converging motions can show up more explicitly than shearing motions due to flux cancellation on magnetograms. This may lead one to consider converging motions to be a more general way of increasing magnetic shear. When a magnetic field evolves in response to a footpoint motion in the ideal MHD regime starting with a certain field configuration, the final field configuration does not depend on the path of moving footpoints as long as the final footpoint positions are the same for all cases with different paths. This, however, is not true when magnetic reconnection is allowed because field line connectivity can be varied by the history of the footpoint motion. We thus examine how flaring phenomena differ for different footpoint paths.

Another interesting question is how a magnetic arcade system containing a magnetic island will evolve when the magnetic shear of the the arcades surrounding (overlying and underlying) the island is reduced. Of course, we do not expect the process leading to the island formation to be exactly reversed because magnetic reconnection is a dissipative, irreversible process. In a philosophical view, however, a change in the global field topology may have analogy with a phase transition in a thermodynamic system. A reversal of the global field topology may take place if we extract magnetic energy out of the system. In this paper, we thus investigate the evolution of a magnetic arcade-island complex system under shear-reducing footpoint motion. This study may 
be relevant to sudden disappearance or "disparition brusque" of a prominence not associated with a solar eruptive event.

In short, this paper reports our investigation on the long-term evolution of a bipolar active region subject to various field line footpoint motions. The results are used to explain the mechanism of homologous flares and physical processes involved in general flaring phenomena. In $\S 2$, a mathematical description of sheared magnetic arcades is presented. The modeling and numerical procedure are expounded in $\S 3$. In $\S 4$, the resistive evolution of a magnetic arcade under a persisting shearing motion is investigated. Section 5 deals with converging footpoint motions imposed to a sheared arcade and also a combination of converging and shearing motions simultaneously imposed. In $\S 6$, the evolution of a sheared arcade-island system under a shear-reducing footpoint motion is studied. A summary and discussion are given in $\S 7$.

\section{MATHEMATICAL DESCRIPTION OF SHEARED MAGNETIC ARCADES}

In this study, we investigate the evolution of $2-1 / 2 \mathrm{D}$ bipolar magnetic arcades in a Cartesian coordinate system. The magnetic arcade occupies the half-space $\{y>0\}$ and the plasma and magnetic field quantities are assumed to be invariant in $z$. The solar surface is modeled by the $x z$-plane $(\{y=0\})$ ignoring the curvature, and the polarity inversion line lies along the $z$-axis $(\{x=0, y=0\})$ as depicted in Figure 1. It is supposed that the magnetic field is potential at $t=0$ and then evolves in response to the plasma flows in the solar surface for $t>0$. For simplicity, but without significant loss of generality, we assume that the initial field is symmetric across the $y z$-plane $(\{x=0\})$ and that the boundary flows on the plane $\{y=0\}$ are antisymmetric across the $z$-axis and invariant in $z$. Then, all the physical variables appearing in magnetohydrodynamic (MHD) description of plasma preserve a symmetry or an antisymmetry across the $y z$-plane as well as invariance in the $z$-coordinate for all the time.

The invariance in $z$ in the $2-1 / 2 \mathrm{D}$ system and the Gauss law, $\nabla \cdot \mathbf{B}=0$, allow us to express the magnetic field with only two scalar variables as

$$
\mathbf{B}=\hat{\mathbf{z}} \times \nabla \psi+B_{z} \hat{\mathbf{z}},
$$

where the poloidal flux function $\psi$ is related to the the $z$-component of the vector potential by $\psi=-A_{z}$. Because $\mathbf{B} \cdot \nabla \psi=0$, a magnetic field line lies in a constant $\psi$ surface. In equilibrium, one can show that $\mathbf{B} \cdot \nabla B_{z}=0$, which means that the toroidal field $B_{z}$ is constant along the field line (see e.g., Cheng \& Choe 1998). This is a good approximation in a quasi-static evolution.

In this study, we deal with a bipolar magnetic arcade such that

$$
B_{y}(x, y=0)= \begin{cases}\geq 0 & \text { if } x<0 \\ \leq 0 & \text { if } x>0\end{cases}
$$

and the maximum of $\psi$ is located at $x=0, y=0$. In such a field, two values of $x$ corresponds to one value of $\psi$ on the $\{y=0\}$ plane, i.e., $\psi(x)$ is a single-valued function whereas $x(\psi)$ has two branches: 
$x^{+}(\psi)>0$ and $x^{-}(\psi)<0$. If a symmetry is assumed across the $\{x=0\}$ plane, $x^{-}(\psi)=-x^{+}(\psi)$. Due to the invariance in $z$, a field line of a potential magnetic field lies in a plane parallel to the $x y$-plane. We now impose on a potential field a shearing boundary flow $V_{z}(x, t)=v_{z}(x, y=0, t)$ parallel to the polarity inversion line. We define $\zeta(x, t)$ as the displacement in the $z$-direction of the fluid element at the boundary location $x$ generated by this flow (see Fig. 1), i.e.,

$$
\zeta(x, t) \equiv \int_{0}^{t} V_{z}\left(x, t^{\prime}\right) d t^{\prime},
$$

and denote by $\Delta \zeta(\psi)$ the difference of $\zeta$ between two conjugate footpoints located at $x^{+}(\psi)$ and $x^{-}(\psi)$, i.e.,

$$
\Delta \zeta(\psi) \equiv \zeta\left(x^{+}\right)-\zeta\left(x^{-}\right)
$$

On the other hand, the distance in the $z$-direction between the two conjugate footpoints of a field line labeled by $\psi$ is given by

$$
\Delta Z(\psi) \equiv Z\left(x^{+}\right)-Z\left(x^{-}\right)=\int_{\psi} \frac{B_{z}}{|\nabla \psi|} d s_{p},
$$

where $Z(x)$ is the $z$-coordinate of the footpoint at $x$ of the field line labeled by $\psi$. The above integration is performed along the field line designated by $\psi$ and $s_{p}$ is the arclength of the field line projected on the $x y$-plane. If the plasma occupying the whole space is a perfect conductor and if there is no reconnection, the distance in the $z$-direction between two conjugate field line footpoints is the same as the sum of the magnitudes of the plasma displacement at $x^{+}$and at $x^{-}$, i.e.,

$$
\Delta Z(\psi)=\Delta \zeta(\psi)
$$

Assuming an antisymmetric shearing motion such that $V_{z}(-x, t)=-V_{z}(x, t)$ and defining $Z(\psi) \equiv$ $(1 / 2) \Delta Z(\psi)$ and $\zeta(\psi) \equiv \zeta\left[x^{+}(\psi)\right]$, equation $(6)$ can be rewritten as

$$
Z(\psi)=\zeta(\psi)
$$

The toroidal flux ( $B_{z}$ flux) through an area surrounded by the flux surface labeled by $\psi$ and the plane $\{y=0\}$ is

$$
\Phi_{z}(\psi)=\int_{\psi^{\prime}=\psi}^{\psi^{\prime}=\psi_{o}}\left(\int_{\psi^{\prime}} \frac{B_{z}}{\left|\nabla \psi^{\prime}\right|} d s_{p}\right) d \psi^{\prime}
$$

where $\psi_{o}=\psi(x=0, y=0)$. By differentiating this equation, we obtain the toroidal flux ( $B_{z}$ flux) through the area made by the unit poloidal flux around the field line labeled by $\psi$. This quantity will be called the differential $B_{z}$ flux at $\psi$ and denoted by $\phi_{z}(\psi)$;

$$
\phi_{z}(\psi)=-\frac{d \Phi_{z}(\psi)}{d \psi}=\int_{\psi} \frac{B_{z}}{|\nabla \psi|} d s_{p} .
$$

Comparing this with equation (5), we can see that

$$
\phi_{z}(\psi)=\Delta Z(\psi) .
$$


Thus, the distance in the $z$-direction between two conjugate footpoints is nothing but the differential $B_{z}$ flux. When there exists diffusion of magnetic fields, the differential $B_{z}$ flux is not conserved even if the plasma at the boundary does not move. This means a slippage of field lines with respect to the plasma elements. Thus, equations (6) and (7) do not hold under magnetic diffusion. The discrepancy between $\Delta Z(\psi)$ and $\Delta \zeta(\psi)$ is negligible in a system with a very small resistivity such as the solar atmosphere unless there is magnetic reconnection. However, a magnetic reconnection process connects a field line segment with another, which was originally not connected to the former, resulting in a considerable discrepancy between $\Delta Z(\psi)$ and $\Delta \zeta(\psi)$.

When magnetic reconnection is allowed in a magnetic arcade, a magnetic island can be created by reconnection and there can be more than one flux surface labeled by $\psi$. In this case, the total differential $B_{z}$ flux at $\psi$ can be defined as the sum of the differential $B_{z}$ fluxes for every flux surface labeled by the same $\psi$, i.e.,

$$
\phi_{z}(\psi)=\sum_{i} \phi_{z i}(\psi)=\sum_{i} \Delta Z_{i}(\psi)=\sum_{i} \int_{\psi} \frac{B_{z}}{|\nabla \psi|} d s_{p i},
$$

where the index $i$ designates the individual field line labeled by the same $\psi$. In other words, the total differential $B_{z}$ flux at $\psi$ is the sum of the footpoint distance in the $z$-direction for the field line $\psi$ connected to the boundary and the pitch of the helical field line $\psi$ in a magnetic island for one rotation in the poloidal plane. Ideally, we may think of pointwise magnetic reconnection with a diffusion region of a zero volume. In this pointwise reconnection process, any quantities contained in a flux tube are conserved. Only in this ideal case, it holds that

$$
\Delta \zeta(\psi)=\sum_{i} \Delta Z_{i}(\psi)=\phi_{z}(\psi)
$$

and under the symmetry across the $y z$-plane

$$
\zeta(\psi)=\sum_{i} Z_{i}(\psi)=\frac{1}{2} \phi_{z}(\psi)
$$

In reality, a reconnection region has a finite volume however small it may be and $\phi_{z}(\psi)$ is not conserved through reconnection. It rather diverges at the separatrices connected to the X-line because the differential flux volume (area in 2D), defined as the flux tube volume per unit poloidal flux such that

$$
\mathcal{S}(\psi)=\int_{\psi} \frac{d s_{p}}{|\nabla \psi|}
$$

generally diverges at the separatrices (cf. Vekstein \& Priest 1992, for possible exceptions) while $B_{z}$ is not zero even on the X-line (Choe \& Lee 1996b). Away from the separatrix, however, equations (12) and (13) hold approximately in a small resistivity system (Choe \& Lee 1996b). This implies that a reconnection process redistributes the differential $B_{z}$ flux, which was contained in an arcade flux tube, into two different flux volumes: one in a new arcade and the other in a magnetic island. 
In this study, we do not only deal with boundary flows parallel to the $z$-axis, but also with boundary flows converging towards or diverging from the polarity inversion line parallel to the $x$-axis. We thus define the distance between the field line footpoints in the $x$-direction as

$$
\Delta X(\psi)=x^{+}(\psi)-x^{-}(\psi) \text {. }
$$

Assuming, for simplicity, that the boundary flows to be antisymmetric across the $y z$-plane, i.e., $v_{x}(x, y=0)=-v_{x}(-x, y=0)$, we have $\Delta X(\psi)=2\left|x^{ \pm}(\psi)\right|$. Thus, we define the perpendicular distance of a footpoint of a field line labeled by $\psi$ from the polarity inversion line as (see Fig. 1)

$$
\xi(\psi)=\left|x^{ \pm}(\psi)\right|
$$

The field lines of a potential field, in which $\zeta=0$, do not have a $z$-component. A footpoint displacement in the $z$-direction makes the field line tilt toward the $z$-direction. The tilt of a field line toward the $z$-direction from the $x$-direction is commonly called "magnetic shear" although there are several quantitative ways of defining magnetic shear (Hagyard et al. 1984; Wang 1992; Lü, Wang, \& Wang 1993). The magnetic shear increases with $|Z(\psi)|$ and decreases with $\xi(\psi)$. The quantity

$$
\bar{Z}(\psi)=\Delta Z(\psi) / \Delta X(\psi)=Z(\psi) / \xi(\psi),
$$

which will be called the relative footpoint displacement, can be a measure of magnetic shear. This quantity is most relevant when comparing evolutions of magnetic arcades of different sizes or when dealing with the arcade evolution with contraction or expansion of the boundary flux distribution. To illustrate this point, we consider a $2-1 / 2 \mathrm{D}$ force free field, which is a good approximation of a quasi-static state in a low $\beta$ plasma, described by

$$
\nabla^{2} \psi+\frac{d}{d \psi}\left(\frac{B_{z}^{2}}{2}\right)=0
$$

If a certain flux function $\psi(x, y)$ and a toroidal field $B_{z}(\psi)$ satisfy the above equation, one can show that

$$
\tilde{\psi}(x, y)=\psi(k x, k y)
$$

and

$$
\tilde{B}_{z}(\tilde{\psi})=k B_{z}(\tilde{\psi})
$$

also satisfy equation (18). The transform given by equation (19) means a self-similar contraction (if $k>1$ ) or expansion (if $k<1$ ) of the poloidal field configuration by a constant scale $k$. Under this contraction (or expansion), the differential flux volume $\mathcal{S}$ defined by equation (14) becomes $1 / k^{2}$ of the original volume. The equilibrium condition given by equation (20) implies that the differential $B_{z}$ flux should be $1 / k$ of the original value, i.e.,

$$
\tilde{\phi}_{z}(\tilde{\psi})=\phi_{z}(\tilde{\psi}) / k
$$

because $\phi_{z}=B_{z} \mathcal{S}$. One can also easily see that the total magnetic energy in the halfspace $\{y>0\}$ is invariant under this transform. Consequently, as far as a $2-1 / 2 \mathrm{D}$ force-free field without an 
island is concerned, a self-similar contraction (or expansion) with invariant $\Delta Z(\psi) / \Delta X(\psi)$ does not change the equilibrium configuration. If the boundary flux distribution contracts (expands) without varying the $z$-coordinate of the footpoint, it is equivalent to increasing (decreasing) the footpoint displacement in the $z$-direction without changing the boundary flux distribution. In our simulation, we impose velocity vectors at the boundary as boundary conditions and have control of $\zeta(\psi)$ rather than $Z(\psi)$, which comes out from the solution. Thus, we define a controllable quantity $\bar{\zeta}(\psi)$ as

$$
\bar{\zeta}(\psi)=\zeta(\psi) / \xi(\psi),
$$

which we will call the relative plasma displacement.

It is useful to comment that the $2-1 / 2 \mathrm{D}$ geometry has a few known disadvantages for realistic modeling of the solar atmosphere. First, field line opening is energetically impossible and the expulsion of a magnetic island to a long distance is also more difficult than in a spherical geometry (Mikić \& Linker 1994; Choe \& Lee 1996b). Second, a much larger footpoint displacement is required than in 3D models to create the same amount of shear angle, which is defined as the angle between the transverse component vector of the sheared field and that of the potential field. The 3D model of a sheared field by Antiochos, Dahlburg, \& Klimchuk (1994) even shows a shear angle larger than $90^{\circ}$. With all these shortcomings, a model in a 2-1/2D Cartesian geometry can still be justified, not merely by the ease in its development and interpretation, but by its restraining character; in other words, any violent behavior showing up in this model will take place in models with a more realistic geometry.

\section{DESCRIPTION OF MODELING}

\subsection{Equations Governing Resistive MHD}

The equations governing the evolution of our model corona are a full set of $2-1 / 2 \mathrm{D}$ MHD equations including gravity and resistivity as follows:

$$
\begin{gathered}
\frac{\partial \rho}{\partial t}+\nabla \cdot(\rho \mathbf{v})=0, \\
\rho\left(\frac{\partial \mathbf{v}}{\partial t}+\mathbf{v} \cdot \nabla \mathbf{v}\right)=\mathbf{J} \times \mathbf{B}-\nabla p+\rho \mathbf{g}+\nabla \cdot \mu \nabla \mathbf{v}, \\
\frac{\partial \psi}{\partial t}+\mathbf{v} \cdot \nabla \psi=\eta \nabla^{2} \psi, \\
\frac{\partial B_{z}}{\partial t}=\frac{\partial}{\partial x}\left(v_{z} B_{x}-v_{x} B_{z}\right)+\frac{\partial}{\partial y}\left(v_{z} B_{y}-v_{y} B_{z}\right)+\nabla \cdot\left(\eta \nabla B_{z}\right), \\
\frac{d}{d t}\left(\frac{p}{\rho^{\gamma}}\right)=0, \\
p=\rho R T,
\end{gathered}
$$




$$
\begin{aligned}
B_{x} & =-\frac{\partial \psi}{\partial y}, \\
B_{y} & =\frac{\partial \psi}{\partial x}, \\
\mathbf{J} & =\nabla \times \mathbf{B},
\end{aligned}
$$

where all the quantities are expressed in a nondimensionalized form by a proper normalization. The magnetic field $\mathbf{B}$ is normalized by $B_{0}$ the maximum magnitude of the boundary normal field, the mass density $\rho$ by the initial density $\rho_{0}$ at the bottom boundary, the velocity $\mathbf{v}$ by $v_{0}=$ $B_{0} /\left(4 \pi \rho_{0}\right)^{1 / 2}$, the time $t$ by $t_{0}=L_{0} / v_{0}$, and the resistivity $\eta$ by $L_{0} v_{0}$, in which $L_{0}$ is the length unit. The normalization units are listed in table 1 . To treat the energetics of the solar corona properly, we need to consider the coronal heating, radiative cooling and anisotropic heat conduction (e.g., Choe \& Lee 1992). However, without a well established knowledge in the coronal heating mechanism, we adopt a polytropic relation (eq. [27]) instead of a full energy equation. Even with this simplification, it is not easy to determine the polytropic index $\gamma$. Considering the high thermal conductivity in the corona, we just set $\gamma=1$ assuming an isothermal atmosphere with temperature of $2 \times 10^{6} \mathrm{~K}$. The gravity $\mathbf{g}=-g \hat{\mathbf{y}}$ is a function of $y$ given by

$$
g(y)=\frac{4 \pi G M_{\odot}}{\left(R_{\odot}+y\right)^{2}}=g_{0} \frac{R_{\odot}^{2}}{\left(R_{\odot}+y\right)^{2}},
$$

where $G$ is the gravitational constant, $M_{\odot}$ the solar mass, $R_{\odot}$ the solar radius and $g_{0}=2.74 \times$ $10^{4} \mathrm{~cm} \mathrm{~s}^{-2}$ is the surface gravity. In the coronal dynamics without a prominence, the gravitational force is not so important compared to Lorentz forces, but it is included in our formulation because without the gravity the plasma $\beta$ would become unrealistically large in the upper part of the computational domain. In our simulation, a constant kinematic viscosity $\nu=\mu / \rho=10^{-3}$ is used for the purpose of numerical smoothing.

\subsection{Initial and Boundary Conditions}

In this study, we assume that the magnetic field is initially potential and the atmosphere is initially in a hydrostatic equilibrium. The boundary flux profile for a bipolar arcade we impose at $y=0$ is chosen as

$$
\psi(x, y=0, t=0)=\frac{8}{(x / a)^{2}+3},
$$

which can be generated by a dipole located at $x=0, y=-\sqrt{3} a$. The corresponding normal magnetic field profile at the bottom boundary is given by

$$
B_{y}(x, y=0, t=0)=-\frac{1}{a} \frac{16(x / a)}{\left[(x / a)^{2}+3\right]^{2}} .
$$

Note that $B_{y}(x, y=0) \geq 0$ for $x \leq 0,<0$ for $x>0$ and that the $\left|B_{y}\right|$ maximum is located at $x= \pm a$ with a value of $1 /$ a. Figure 2 a shows the $\psi$ and $B_{y}$ profiles for $a=1$. By solving a Laplace 
equation $\nabla^{2} \psi=0$ with the boundary conditions of equation (33) and $\psi=0$ at infinity, we have

$$
\psi(x, y, t=0)=\left(\frac{8}{\sqrt{3}}\right) \frac{(y / a)+\sqrt{3}}{(x / a)^{2}+(y / a+\sqrt{3})^{2}} .
$$

The tangential magnetic field profile at the bottom boundary at $t=0$ is given by

$$
B_{x}(x, y=0, t=0)=-\frac{8}{\sqrt{3} a} \frac{(x / a)^{2}-3}{\left[(x / a)^{2}+3\right]^{2}} .
$$

It is to be noted that the $\left|B_{x}\right|$ maximum is located at $x=0$ and that $B_{x}$ changes sign at $x= \pm \sqrt{3} a$.

The photosphere modeled by the bottom boundary is considered as a perfect conductor such that the magnetic field is frozen into the plasma and no magnetic fields above the model photosphere can diffuse into it. Assuming no flows across the bottom boundary, the flux of the boundary normal magnetic field is conserved. This also implies the conservation of the poloidal flux in the simulation domain because the $\psi$ maximum, $\psi_{0}$, is located at the origin where the flow velocity is zero. We impose the tangential velocity fields at the bottom boundary as described in $\S 3.3$ and the evolution of flux function $\psi(x, y=0, t)$ is governed by the perfect conductor condition

$$
\frac{\partial \psi}{\partial t}=-v_{x} \frac{\partial \psi}{\partial x}
$$

The toroidal magnetic field $B_{z}$ at the bottom boundary is determined by implementing equation (26) in a half-size cell contiguous to the boundary. The density $\rho$ at the bottom boundary is set to be constant in time and equal to the initial value. Since this setting is an overspecification of the boundary condition, an unresolvable boundary layer appears at the bottom boundary. However, the effect of this boundary layer in the global dynamics is minimal because the plasma $\beta$, the ratio of plasma pressure to magnetic pressure, is very low in the neighborhood of the bottom boundary $\left(\beta \sim 2.5 \times 10^{-3}\right.$ at the origin). This boundary condition rather helps keep the time step size from getting too small due to the rarefaction of plasma. A further discussion can be found in Choe \& Lee (1996a).

The boundary conditions at $x=0$ are determined by the symmetry property. The other lateral boundary and the upper boundary are assumed to be open and this condition is approximately implemented with the zeroth order extrapolation except for $\psi$, i.e.,

$$
\frac{\partial F_{i}}{\partial n}=0
$$

in which the derivative is taken in the direction normal to the boundary and $F_{i}$ respectively stands for $\mathbf{v}, B_{z}$ and $\rho^{n+1}-\rho^{n}$, where the superscripts denote the time step. Since the flux function $\psi$ labels each field line moving frozen in the plasma element in ideal MHD, we impose the following boundary condition at those two open boundaries:

$$
\frac{\partial \psi}{\partial t}=-\left(\mathbf{v}_{n}^{*}+\mathbf{v}_{t}\right) \cdot \nabla \psi
$$


where $n$ and $t$ respectively denote the boundary normal and tangential components and

$$
\mathbf{v}_{n}^{*}=\left\{\begin{array}{ll}
\mathbf{v}_{n} & \text { if } \mathbf{v} \cdot \hat{\mathbf{n}}>0 \\
\mathbf{0} & \text { if } \mathbf{v} \cdot \hat{\mathbf{n}} \leq 0
\end{array},\right.
$$

where $\hat{\mathbf{n}}$ is the outward normal unit vector.

\subsection{Tangential Velocity Profiles at the Bottom Boundary}

At the bottom boundary, the shearing velocity $v_{z}$ is given as a function of the $x$-coordinate and time when there is no diverging or converging motion (Case 1 where $a=1$ ), i.e.,

$$
v_{z}(x, y=0, t)=f_{z}(t) V_{z}(x),
$$

where

$$
V_{z}(x)=V_{z 0} x \exp \left(\frac{1-x^{2}}{2}\right)
$$

whose profile is shown in Figure $2 \mathrm{~b}$. The time dependent part $f_{z}(t)$ determines the duration of the shearing motion and its acceleration and deceleration periods, i.e.,

$$
f_{z}(t)= \begin{cases}\left(t-\tau_{0}\right) /\left(\tau_{1}-\tau_{0}\right) & \text { if } \quad \tau_{0} \leq t<\tau_{1} \\ 1 & \text { if } \quad \tau_{1} \leq t<\tau_{2} \\ \left(t-\tau_{2}\right) /\left(\tau_{f}-\tau_{2}\right) & \text { if } \quad \tau_{2} \leq t<\tau_{f} \\ 0 & \text { otherwise }\end{cases}
$$

In the cases that footpoints also move in the $x$-direction, the shearing velocity $V_{z}$ is not given as a function of $x$, but as a function of $\psi$ by converting the independent variable in equation (41) into $\psi$ with the use of equation (33). That is,

$$
V_{z}(\psi)= \pm\left[e\left(\frac{8}{\psi}-3\right)\right]^{\frac{1}{2}} V_{z 0} \exp \left[-\frac{1}{2}\left(\frac{8}{\psi}-3\right)\right],
$$

where the sign is the same as that of $x$. The prescription of the shearing velocity as a function of each field line is adopted in order to facilitate the computation of $\zeta(\psi)$ and $\bar{\zeta}(\psi)$ and compare on good grounds the field evolutions having undergone different footpoint paths. As can be seen in equation (41), the boundary normal field maxima are subject to the highest shearing velocity. The fluid elements at those locations thus travel in the $z$-direction the greatest distance from the initial locations and this distance, denoted by $\zeta_{m}(t)$, is

$$
\zeta_{m}(t)=V_{z 0} \int_{0}^{t} f_{z}\left(t^{\prime}\right) d t^{\prime}
$$

To consider a converging or diverging velocity field that does not indefinitely accumulate field lines or mass anywhere within a finite time, we choose

$$
v_{x}(x, y=0, t)=f_{x}(t) V_{x}(x),
$$


where $V_{x}(x)$ is linear in $x$ up to a certain distance $x_{L}$ from the polarity inversion line and then gradually decreases with $|x|$, i.e.,

$$
V_{x}(x)=\left\{\begin{array}{lll} 
\pm V_{x 0} \frac{x}{x_{L}} & \text { if } & |x| \leq x_{L} \\
\pm V_{x 0} \frac{x}{x_{L}} \exp \left[-\frac{\left(x-x_{L}\right)^{2}}{x_{L}^{2}}\right] & \text { if } & |x|>x_{L}
\end{array}\right.
$$

in which the positive sign refers to a diverging flow and the negative sign to a converging flow. A converging velocity profile given by equation (46) for $x_{L}=6 a=18$ is shown in Figure 2c. The time-dependent function $f_{x}(t)$ is prescribed similarly to $f_{z}(t)$. The solution of equation (37) for $|x| \leq x_{L}$ is given by

$$
\psi(x, t)=\psi\left(\frac{x}{\vartheta(t)}, 0\right)
$$

where

$$
\vartheta(t)=\exp \left( \pm \frac{V_{x 0}}{x_{L}} \int_{0}^{t} f_{x}(t) d t\right)
$$

For converging flows, a negative sign gives $1 \geq \vartheta(t)>0$ and for diverging flows, a positive sign gives $1 \leq \vartheta(t)<\infty$. Equation (47) implies a self-similar contraction or expansion of the boundary normal flux profile, and the footpoint positions $x^{ \pm}(\psi)$ vary in the same ratio for all the field lines in $|x|<x_{L}$, i.e.,

$$
x(\psi, t)=\vartheta(t) x(\psi, 0) .
$$

Now, the distance of a boundary normal field maximum from the polarity inversion line at time $t$ is denoted by $\xi_{m}(t)$. Since $\xi_{m}(t=0)=a$ from equation (34) and $a<x_{L}$, we have

$$
\xi_{m}(t)=\vartheta(t) \xi_{m}(t=0)=a \vartheta(t) .
$$

The relative plasma displacement (eq. [22]) at the boundary normal field maxima, which will be denoted by $\bar{\zeta}_{m}(t)$, is thus

$$
\bar{\zeta}_{m}(t)=\frac{\zeta_{m}(t)}{\xi_{m}(t)}
$$

\subsection{Numerical Procedures}

Our computational domain consists of a rectangular area $\{(x, y) \mid 0 \leq x \leq 100,0 \leq y \leq 150\}$ and is covered by a nonuniform grid with $127 \times 181$ mesh points, in which the ratio of the smallest grid size to the largest in each direction is 1 to 40 . The governing equations are finite-differenced and integrated in time employing a semi-implicit scheme (e.g., Harned \& Schnack 1986), which allows 4-10 times as large a time step size as constrained by the CFL (Courant-Friedrichs-Lewy) condition. For a detailed account of the numerical algorithm, readers are referred to Choe \& Lee (1992). 
We have run many simulation cases with different footpoint motion patterns and different values of resistivity and here report results of nine selected cases as listed in table 2. All Case 1's involve only shearing footpoint motions parallel to the polarity inversion line. In Case 2's, a converging footpoint motion is imposed after a shearing footpoint motion is first imposed and then stopped. Both shearing and converging motions are simultaneously given in Case 3. A diverging footpoint motion is applied to a pre-sheared arcade in Case 4.

For the case either considering only a shearing motion (Case 1 in which $v_{z} \neq 0, v_{x}=0$ at $y=0$ ) or involving a diverging motion (Case 4 in which $v_{x}(x>0, y=0)>0$ ), we set $a=1$ so that the $\left|B_{y}\right|$ maximum is initially located at $x= \pm 1$ with a value of unity. For Case 4 , we also set $x_{L}=18$ for the diverging motion profile (see eq. [46]). The cases involving converging motions, Cases 2 and 3, are set up with a more dispersed initial boundary normal flux profile, i.e., $a=\xi_{m}(t=0)=3$ in equations (33) and (34) in order to maintain a proper spatial resolution near the origin even after field line densities are much increased there. The converging motion profile is chosen with $x_{L}=18$ so that footpoints in at least $92 \%$ of the total magnetic flux may be under self-similar evolutionary motion.

Subcases in each of these four cases are differentiated by the value of resistivity. Since our simulation code has an intrinsic numerical diffusion corresponding to $\eta \sim 10^{-6}$, resistive effects

for $\eta<5 \times 10^{-6}$ cannot be properly resolved. Thus, to obtain information about the case with $\eta=2 \times 10^{-6}$, we run a case (Case 1C) with $\eta=10^{-5}$ and with a shearing velocity five times as large as that in other subcases in Case 1. This substitution can be justified by the fact that the shearing speed is much smaller than the Alfvén speed in the system.

\section{REPETITIVE FLARING IN CONTINUOUSLY SHEARED MAGNETIC ARCADES: CASE 1}

In this section, we consider only shearing footpoint motions parallel to the polarity inversion line. As shown in table 2, four simulation runs are performed with different values of resistivity and shearing velocity. All the simulation runs show similar evolutionary features except for the case with zero resistivity (Case 1D). A typical evolutionary trend is well demonstrated in Figure 3 which shows field lines at different times in Case $1 \mathrm{~A}\left(\eta=10^{-5}\right.$ and $\left.V_{z 0}=10^{-3} v_{0}\right)$. As the magnetic shear is increased by the shearing footpoint motion, the current layer in the center of the magnetic arcade becomes thinner in the $x$-direction and longer in the $y$-direction so that magnetic reconnection takes place and a magnetic island is created. This result was reported by previous numerical simulation studies (Mikić \& Linker 1994; Choe \& Lee 1996b; Amari et al. 1996a). The magnetic reconnection is found to be triggered around $t \approx 11000 t_{0}$ which corresponds to $\zeta_{m} \approx 11$. The existence of a magnetic island in a low $\beta$ plasma is conditioned by the presence of the toroidal magnetic field ( $B_{z}$ in our notation) in it. The magnetic reconnection divides and redistributes the toroidal flux previously contained in line-tied flux tubes into two new flux systems: one with the magnetic island and the other with the line-tied reconnected field. The differential toroidal flux 
in a reconnected field line is thus less than the value before the reconnection. In other words, the magnetic shear in the arcade under the magnetic island is reduced because the reconnected field lines in the underlying arcade have a smaller conjugate footpoint distance in the $z$-direction than the old field lines before magnetic reconnection occurs. Although the conjugate footpoint distance may not be defined and diverge in the separatrix connected to the X-line (Choe \& Lee 1996b), the toroidal flux in a finite flux volume does not diverge in any case and the magnetic shear in the underlying arcade is always decreased after reconnection. By a continuing shearing motion, the magnetic shear in the underlying arcade is again increased and a new reconnection is initiated at $t \approx 19000 t_{0}\left(\zeta_{m} \approx 19\right)$. The new magnetic island so created rises and pushes up the line-tied field lines surrounding it so that these line-tied field lines start to reconnect with the field lines in the upper island through the upper X-line. After all the line-tied field lines in the underlying arcade surrounding the newborn island have reconnected with the upper island field lines, the two magnetic islands quickly coalesce to form one island. This merged island keeps on rising as field lines surrounding it continue to reconnect in the vertical current sheet below and it gains flux. The magnetic reconnection to generate a new island, the newborn island's merging with an upper island and the reconnection under the integrated island are considered to constitute a flaring event. Figure 3 shows that the generation of a new island and the subsequent merging of islands are repeated with some time interval while the shearing footpoint motion continues. This repetitive occurrence of a sequence of reconnection processes is interpreted as a set of homologous flares. For Case $1 \mathrm{~A}$, new magnetic islands are created at $t \approx 11000 t_{0}\left(\zeta_{m} \approx 11\right), 19000 t_{0}\left(\zeta_{m} \approx 19\right), 29000 t_{0}$ $\left(\zeta_{m} \approx 29\right)$ and $40000 t_{0}\left(\zeta_{m} \approx 40\right)$, respectively. The island merging occurs in a relatively short time after a new island is born. The time interval between successive creation of new islands is thus around $10000 t_{0}$, and with $t_{0}=9.3 \mathrm{~s}$ (table 1 ) it is about a day, although it has a mild tendency of increase with progress in island generation.

To investigate kinematics of island systems, the height of O-lines in the islands versus time is shown in Figure 4. Compared with the first island and other integrated islands, newborn islands rise far faster. For example, the rising speed of the integrated island formed at $t \approx 20000 t_{0}$ is about $1.3 \times 10^{-3} v_{0}$ (with $v_{0}=3.2 \times 10^{3} \mathrm{~km} \mathrm{~s}^{-1}$, this speed is about $4 \mathrm{~km} \mathrm{~s}^{-1}$ ). On the other hand, the new island born at $t \approx 29000 t_{0}$ rises in the beginning at a speed of $\sim 3 \times 10^{-3} v_{0}$ (about $10 \mathrm{~km} \mathrm{~s}^{-1}$ ) and in the merging stage at a speed of $\sim 2 \times 10^{-2} v_{0}$ (about $65 \mathrm{~km} \mathrm{~s}^{-1}$ ). With the shift in island generation, the integrated island as well as the newborn island gets faster. From other simulation runs with different resistivity, we have found that the rising speed of islands also depends on the reconnection rate. With resistivity of $5 \times 10^{-5}$, the rising speeds of newborn islands are more than twice of those in Case $1 \mathrm{~A}\left(\eta=10^{-5}\right)$ and with $\eta=10^{-3}$ they are almost ten times those in Case $1 \mathrm{~A}$. The rising speed of a newborn island in a merging process is roughly proportional to $\eta^{1 / 2}$. However, it should be kept in mind that this result is obtained with a spatially uniform resistivity. As shown in previous MHD simulations (Choe \& Lee 1996b), the reconnection rate can vary depending on the size of the diffusion region and the spatial profile of resistivity. Therefore, one should not give too much meaning to the above numbers, but should pay attention to the relative magnitudes. 
The merging of two islands is a natural consequence because the toroidal currents $\left(J_{z}\right)$ of both islands are in the same direction and thus attract each other. In Figure 4, we should note that the motion of newborn islands comprises two phases; the island rises rather slowly, although faster than the pre-existing one, in the first phase and then much faster in the second phase. This behavior is similar to the simulation results on the coalescence instability by Biskamp \& Welter (1980), in which they also identified two phases during the island coalescence. The first phase mainly involves an ideal MHD process leading to field compression and current sheet thinning. Dynamics in the second phase is mostly governed by magnetic reconnection between the upper island and the underlying flux system containing a newborn island. The rising speed of the newborn island thus depends on the reconnection rate. As can be noticed in the motion of the first island created at $t \approx 11000 t_{0}\left(\zeta_{m} \approx 11\right)$, a single island does not have the second phase with a faster motion. Although the altitude of the integrated island becomes higher and higher with time, it has moved only a few solar radii in a few days in our simulation. This is because the 2D Cartesian geometry adopted in our simulation energetically inhibits the island system from totally escaping from the sun. In a more realistic $3 \mathrm{D}$ geometry, however, we expect the island to be accelerated more easily and be expelled farther away from the solar surface.

To investigate our simulation results in the aspects of the energy generation and dissipation, we show in Figure 5 the evolution of (a) the ratio of the total magnetic energy to the potential field energy, $W_{\mathrm{B}} / W_{\text {pot }}$, (b) the energy input through the bottom boundary per unit plasma displacement at $x= \pm 1$, which is equivalent to the upward Poynting flux through the bottom boundary normalized with the maximum shearing speed $V_{z 0}$, and (c) the maximum magnitude of the $z$-component of current density, $\left|J_{z}\right|_{\text {max }}$, in the vertically elongated current layer. The total magnetic energy is given by

$$
W_{\mathrm{B}}=\int_{V} \frac{B^{2}}{2} d V
$$

The upward Poynting flux through the bottom boundary is nothing but the energy input rate into the system by the boundary flows and is calculated by

$$
\int_{-\infty}^{\infty} S_{y}(x, y=0) d x=-\int_{-\infty}^{\infty}(\mathbf{v} \times \mathbf{B}) \times \mathbf{B} \cdot \hat{\mathbf{y}} d x=-\int_{-\infty}^{\infty} B_{y} B_{z} v_{z} d x
$$

Because we have cases with different shearing speeds, the above quantities are plotted versus $\zeta_{m}$, the plasma displacement in the $z$-direction at the boundary normal field maxima, rather than versus time. It is for the same reason that we employ as the ordinate of Figure 5b the Poynting flux divided by $V_{z 0}$. In Figure 5, results from four simulation runs (Cases 1A, 1B, 1C and 1D in table 2) with different values of resistivity and shearing speed are displayed. The results for Case 1A with $\eta=10^{-5}$ are shown by solid lines, those for Case $1 \mathrm{~B}$ with $\eta=5 \times 10^{-5}$ are shown by chain-dotted lines, and those for the ideal MHD case (Case 1D) are shown by dotted lines.

As shown in Figure 5a, the energy release by reconnection processes is an increasing function of resistivity. The time interval between the initiation of a new reconnection marked with a filled circle in the figure and the completion of island merging marked with a blank circle is a decreasing 
function of resistivity. These facts just imply that the reconnection rate in our simulation system is an increasing function of resistivity. However, the functional dependence of reconnection rate upon resistivity can vary depending on the spatial profile of resistivity as mentioned earlier. In Case 1C (dashed line), which is nearly equivalent to a case with $\eta=2 \times 10^{-6}$, the energy released by magnetic reconnection is so small that the total magnetic energy is still increasing during flaring events. This means that the energy input by the footpoint motion exceeds the energy dissipation by reconnection in this particular case. Such a case seems improbable in the sun, where the flare energy release rate is much greater than the energy input rate that can be estimated from photospheric observations.

In our simulations, two different types of reconnection processes are involved. The reconnection of line-tied arcade field lines takes place in a vertically elongated current sheet, creating a magnetic island and transferring magnetic fluxes to the island. The reconnection between the upper magnetic island and the underlying flux system containing a newborn island takes place in a horizontally elongated current sheet. This process comprises the reconnection of the upper island with the linetied field and the reconnection between two islands. The sequence of these reconnection processes is regarded to constitute an individual flaring event. Now we investigate the relationship between these reconnection processes and their corresponding meaning in solar flares. The evolution of magnetic energy for Case 1B (chain-dotted line) in Figure 5a most evidently shows that the magnetic energy released by merging of two islands is smaller than the energy released by the subsequent reconnection of line-tied field under the integrated island although the former process proceeds much faster than the latter. Thus, the main phase of a flare can be attributed to the reconnection in a vertically elongated current sheet under the integrated island as in conventional pictures of solar flares (e.g., Sturrock 1968; Kopp \& Pneuman 1976; Tsuneta 1996). Figure 5c shows the maximum current density in the vertically elongated current sheet, $\left|J_{z}\right|_{\text {max }}$. Because the reconnection electric field, given by $E_{z, R e c}=\eta J_{z}$ in the current sheet, equals to the poloidal flux reconnected per unit time, the $\left|J_{z}\right|_{\text {max }}$ curve indicates how much flux is being reconnected in the $\mathrm{X}$-line which is located near the current density maximum. As shown in Figure 5c, the maximum current density increases before the initiation of reconnection in the underlying arcade due to the current sheet thinning, continues to increase during the reconnection processes, peaks at the time of island merging completion, and then slowly decays until a new current sheet is formed in the underlying arcade. The time interval from the reconnection trigger in the underlying arcade and to the end of the slow island rising phase can be interpreted as the preflare phase. The flux reconnecting rate further increases in the fast island rising phase and reaches a maximum when merging of the two islands is completed. This rather short time interval is identified with the impulsive (or flash) phase. The highest flux reconnecting rate in this phase is attributed to the shooting up of the lower island that elongates the line-tied field wrapping around both islands to form a very thin current sheet. After the merging of two islands, reconnection of line-tied field continues, but slows down with decreasing $\left|J_{z}\right|_{\max }$. This phase is longer than the former two phases and is considered as the main phase of a flare. From Figure 5c, we can obtain the reconnection electric field $E_{z, R e c}$. In Case $1 \mathrm{~A}$ $\left(\eta=10^{-5}\right), E_{z, \operatorname{Rec}} \approx 0.64 \mathrm{Vm}^{-1}$ at the time of the second island formation $\left(t \approx 1.9 \times 10^{4} t_{0}\right)$ when 
$\left|J_{z}\right|_{\max } \approx 4.0 J_{0}$ and $E_{z, \text { Rec }} \approx 1.1 \mathrm{Vm}^{-1}$ at the completion of island merging when $\left|J_{z}\right|_{\text {max }} \approx 6.7 J_{0}$. With this electric field, electrons can be accelerated to an energy of $10 \mathrm{keV}$ in $10 \mathrm{~km}$. Because the classical resistivity in the actual solar corona is much lower $\left(\eta \sim 10^{-12}\right)$ than the values used in our simulation, we expect the electric field to be smaller in the actual solar corona. Theories of magnetic reconnection are still being developed and the resistivity scaling of the electric field is still not known. However, if we assume a tearing mode type scaling with the electric field proportional to $\eta^{3 / 5}$, the peak reconnection electric field would be roughly $1.1 \times 10^{-3} \mathrm{Vm}^{-1}$ for an anomalous resistivity of $\eta=10^{-10}$ at the completion of island merging. With this electric field, electrons can be accelerated to an energy of $10 \mathrm{keV}$ in $10,000 \mathrm{~km}$, which is still smaller than a typical flaring arcade size. Thus, high energy electrons responsible for X-ray emission can be generated by the reconnection electric field with our flare mechanism.

In Figure 5, it is noticeable that the time interval between successive flaring events is a decreasing function of resistivity, or in other words, of reconnection rate. In Figure 5b, we can also notice another interesting feature that the energy input rate has an exact anti-correlation with the magnetic energy. The energy input starts to increase when the magnetic energy starts to decrease by a new reconnection event and its maxima coincide with the magnetic energy minima. Also, the overall energy input rate is higher in cases with higher resistivity where magnetic reconnection is more active. Now we address the question how a magnetic reconnection process in the corona is related with the energy input through the solar surface. The upward Poynting vector is expressed as $S_{y}=-B_{z} v_{z} B_{y}$ (eq. [53]) when only a shearing motion is imposed. Since $B_{y}(x, y=0)$ is constant in time and so is $v_{z}(x, y=0) / V_{z 0}$ except for the short initial ramp period, $S_{y} / V_{z 0}$ is most of time proportional to the toroidal magnetic field $B_{z}$. Thus, we have to examine the evolution of $B_{z}$ to understand the evolution of the Poynting flux. When arcade field lines are reconnected forming a magnetic island, the toroidal flux that was held in the line-tied arcade before the reconnection is redistributed into the magnetic island and the reconnected underlying arcade. The magnetic shear (more exactly the differential toroidal flux) is thus reduced in the reconnected line-tied field. Geometrically, the distance between two conjugate footpoints of the reconnected line-lied field line, $\Delta Z(\psi)$, is reduced compared with the footpoint distance of the field line before reconnection because the reconnection process cuts and joins two field line segments from different field lines in the same flux surface. A reduction of the differential toroidal flux $\Delta Z$, however, does not lead to a reduction of the toroidal field $B_{z}$ because the flux volume is also divided by the reconnection process (see eqs. [5] and [14]) and the flux volume of the reconnected line-tied field is smaller than before the reconnection. Therefore, we still do not have enough information to determine whether the toroidal field is increased or decreased by reconnection. In Figure 5b, one can see that in the ideal MHD case without reconnection, Case $1 \mathrm{D}$, the energy input rate increases up to $Z_{m}=\zeta_{m} \approx 2$ and then decreases. Thus, the toroidal magnetic field $B_{z}$ also first increases with the footpoint displacement up to a certain maximum and then asymptotically decreases in an ideal MHD system. The decrease of $B_{z}$ with increasing shear, which may sound strange, is a natural consequence of the fact that the total toroidal magnetic energy has an upperbound (see Aly 1984, 1990; Choe \& Lee 1996a, for detailed expositions). The evolution of an arcade under a magnetic island is, of 
course, different from that of an arcade without an island, but we can reasonably assume that the differential flux volume of an arcade field line would be larger in the ideal MHD case than that of a field line with the same footpoint distance $\Delta Z$ lying under a magnetic island because an arcade is freer to expand without any island above it. Therefore, we can conclude that a reduction of $\Delta Z$ leads to an increase of $B_{z}$ as long as $\Delta Z$ is larger than 4 in our model arcade. Moreover, the arcade under a new magnetic island is dynamically pressed by the reconnection outflows while reconnection is active. Therefore, the toroidal field is increased in the reconnected line-tied field and in consequence, the Poynting flux into the system is enhanced. This feature is also demonstrated in Figure 6, in which the toroidal magnetic fields $\left(B_{z}\right)$ and the upward components of the Poynting vector $\left(S_{y}\right)$ for Case $1 \mathrm{~A}$ are plotted along a segment of the bottom boundary $(0 \leq x \leq 2, y=0)$ for different plasma displacements $\left(\zeta_{m}\right)$ which increases almost proportionally to time. Thick lines are drawn for the time just after the initiation of a new reconnection in the underlying arcade. In the $B_{z}$ plot, it is obvious that the $B_{z}$ curves comprise a high plateau-like part and the descending tail. The plateau-like part encompasses the footpoints of the newly reconnected line-tied field. The break point between the two parts of the curve corresponds to the footpoint of the separatrix that is connected to the new X-line and also envelops the newborn island. With more and more flux reconnected, the footpoint of the separatrix propagates outward. As the reconnection rate decreases, the plateau becomes less prominent. It is also noticed that the tail of the $B_{z}$ curves are again divided into two parts when there exist two magnetic islands. The $S_{y}$ maximum is located near the footpoint of the separatrix because the maximum of our $V_{z}$ profile is located at $x=1$ and the footpoints of new separatrices lie within $|x|<1$. It is also noticed that the value of the Poynting vector increases and decreases in accordance with the activity level of the reconnection process. In conclusion, the more active the magnetic reconnection is, the more energy is generated by a shearing motion and the shorter time is required for accumulation of enough magnetic energy to induce a new magnetic reconnection.

\section{ARCADE EVOLUTION UNDER FOOTPOINT CONVERGENCE: CASES 2 AND 3}

\subsection{Converging Footpoint Motion Imposed on a Pre-sheared Arcade: Case 2}

Now we deal with another type of shear-increasing footpoint motion, i.e., a converging footpoint motion. To increase magnetic shear by a converging motion, we initially need a finite amount of shear, however small it may be. In our simulations, a sheared state is first generated by a pure shearing motion and then a converging motion is imposed. In this case, Case 2, we employ a different initial flux distribution at the bottom boundary from that in Case 1 so that the boundary normal field profile is three times as diffuse a boundary normal field profile as that in Case 1, i.e., $a=\xi_{m}(t=0)=3$ in equations (33) and (34). With this choice, a proper numerical resolution can be maintained after footpoint converging. The shearing velocity profile is also correspondingly diffused as given by equation (43). The maximum shearing speed is set as $V_{z 0}=10^{-3} v_{0}$ and is 
located at the boundary normal field maxima. Including short ramp periods of initial increase and final decrease, the shearing motion persists up to $t=28000 t_{0}$, at which time $\zeta_{m} \approx 27$ and $\bar{\zeta}_{m}=\zeta_{m} / \xi_{m} \approx 9$. This time is chosen so that no magnetic reconnection may take place while the shearing motion is exerted. The converging velocity profile is given by equation (46) with the maximum converging speed $V_{x 0}=10^{-3} v_{0}$ located at $x= \pm x_{L}= \pm 18$. With this boundary velocity profile, the boundary flux profile contracts towards the origin, almost keeping its functional form unchanged. The boundary normal field maxima reach $|x|=1$ at $t \approx 48800 t_{0}$. The three cases reported in this section are differentiated by the resistivity value; $\eta=10^{-5}$ in Case $2 \mathrm{~A}, \eta=5 \times 10^{-5}$ in Case 2B and $\eta=0$ in Case 2C (an ideal MHD case).

In Figure 7, a typical arcade evolution under footpoint convergence is shown for Case 2A. As already known in Inhester et al. (1992) and Lee, Choe, \& Akasofu (1995), magnetic reconnection takes place in the footpoint-converging arcade and a magnetic island is created in our simulation too. The reconnection is triggered at $t \approx 33600 t_{0}$ with $\bar{\zeta}_{m} \approx 12$. This number is similar to that in Case 1A. Different from Case 1's, however, no second island is formed in the underlying arcade in Case 2A until our simulation run ends with the island exit from the computational domain. The same result is also found in cases with different nonzero resistivity values. Now we address the question what makes such a difference between the two types of shear-increasing motion.

In a quasi-static evolution of magnetic field, magnetic reconnection is possible only if the system can evolve into a lower energy state. In other words, the magnetic energy must be increased over a certain threshold in order to trigger magnetic reconnection. We thus look into the energetic evolution of our magnetic arcade to explore the island formation process. Figure 8 shows (a) the magnetic energy and (b) the upward Poynting flux through the bottom boundary versus the relative plasma displacement at the boundary normal field maxima, $\bar{\zeta}_{m}$. Here $\bar{\zeta}_{m}$ is proportional to time while a shearing motion is imposed and increases exponentially with time while a converging motion is going on (see eqs. [48] and [51]) except in short ramp periods. A vertical line is drawn in the plot to indicate the time at which the shearing motion ceases and the converging motion commences. Three different cases are represented by different line drawings: Case $2 \mathrm{~A}$ with $\eta=10^{-5}$ by solid lines, Case $2 \mathrm{~B}$ with $\eta=5 \times 10^{-5}$ by chain-dotted lines and Case $2 \mathrm{C}$ with $\eta=0$ by dotted lines. The magnetic energy evolution for the ideal MHD case (Case 2C displayed in dotted lines) is almost identical with that in Case 1D, the ideal MHD case with a pure shearing motion. This is not surprising because in quasi-static evolutions without topological change in the low $\beta$ limit, the magnetic state at a certain time is entirely determined by specifying the field line footpoint locations. The Poynting flux plot for Case 2C (dotted line in Fig. 8b) looks quite different from that for Case 1D (dotted line in Fig. 5b), but it is due to the different functional dependence upon time of the abscissa in each figure. As in Case 1's (pure shearing motion cases shown in Fig. 5a), more magnetic energy is released with higher resistivity. The sudden decrease of magnetic energy after $\bar{\zeta}_{m} \sim 32$ in Case $2 \mathrm{~B}$ with $\eta=5 \times 10^{-5}$ (chain-dotted line) results from the exit of the magnetic island out of the computational domain. In Case 1's (pure shearing motion cases), the rise and fall of magnetic energy is repeated with repetition of flaring events whereas in Case 
2's (converging motion cases), the magnetic energy continues to decrease after the initiation of magnetic reconnection. This means that the converging motion does not supply enough energy to compensate the energy dissipated by reconnection. Figure $8 \mathrm{~b}$ supports this argument. Contrary to Case 1's (see Fig. 5b) in which the Poynting flux into the system is larger with higher reconnection rate, the Poynting flux in Case 2's is smaller with higher reconnection rate. Thus, among Case 2's, the ideal MHD case (Case $2 \mathrm{C}$ ) produces the largest energy input rate. It is to be noted that the exit of the magnetic island makes the Poynting flux in Case $2 \mathrm{~B}\left(\eta=5 \times 10^{-5}\right)$ larger than that in Case $2 \mathrm{~A}\left(\eta=10^{-5}\right)$ after $\bar{\zeta}_{m} \sim 32$.

The difference in energy input for cases with a shearing motion and cases with a converging motion can be understood by examining the factors constituting the Poynting vector. The $y$ component of the Poynting vector at the bottom boundary subject to a converging motion is given by

$$
S_{y}(x, y=0)=-B_{y}(\mathbf{B} \cdot \mathbf{v})=-B_{y} B_{x} v_{x} .
$$

From this, we see that $S_{y}>0$ only in the boundary parts where field lines are inclined in such a way that we recede from the $\{x=0\}$ plane when we move along a field line from a footpoint upward. If the total poloidal flux is finite, the inner field lines in the neighborhood of the origin are inclined inward and the outer field lines are inclined outward. Thus, the Poynting vector is downward in the inner part of the bottom boundary and it is upward in the outer part. If a shear-increasing footpoint motion, whether a shearing motion or a converging motion, acts on a magnetic arcade without any magnetic island, more and more field lines are inclined outward as the relative footpoint displacement is increased (Choe \& Lee 1996b). When magnetic reconnection takes place in the arcade to form a magnetic island, the footpoint displacement is decreased in the arcade under the island as discussed in $\S 4$. Thus, more field lines are inclined inward at the bottom boundary in a system with an island than in the system without an island that has experienced the same boundary motion. This implies that the upward Poynting flux produced by a converging motion is less in the presence of an island than in the ideal MHD case without an island. Also, the less upward Poynting flux is generated by a converging motion as the more magnetic flux is given to the magnetic island by magnetic reconnection as can be seen in Figure 8b. In Case 2, therefore, the arcade under the island is not supplied with enough energy to induce another reconnection.

\subsection{Shearing and Converging Motions Acting Simultaneously: Case 3}

As seen in the above, shearing motions and converging motions generate different energy input rates in resistive evolutions involving magnetic islands. Now we investigate the evolution of a magnetic arcade subject to shearing and converging motions acting simultaneously. In Case 3 , we begin with an initial boundary normal field profile with $a=\xi_{m}(t=0)=3$ in equations (33) and (34) as in Case 2. The shearing velocity profile is given as a function of flux function $\psi$ by equation (43) and the maximum shearing speed $V_{z 0}$ is chosen to be $10^{-3} v_{0}$ at the boundary normal field maxima. The converging velocity profile is given by equation (46) with the maximum 
converging speed $V_{x 0}=5 \times 10^{-4} v_{0}$ located at $x= \pm x_{L}= \pm 18$. The resistivity is taken to be $\eta=10^{-5}$. Both the shearing and converging footpoint motions are applied from the very beginning of the simulation.

As shown in Figure 9, creation and merging of magnetic islands are repeated in this case as in Case 1. The first reconnection is triggered at $t \approx 38700 t_{0}$ and a new reconnection event in the arcade under the island takes place after a time interval of $\sim 16000 t_{0}$. One may wonder why the first reconnection is initiated at $\bar{\zeta}_{m} \approx 37$, which is much larger than $\bar{\zeta}_{m}$ for reconnection trigger in other cases. This can be understood by referring to the mechanism of reconnection trigger in an arcade-like line-tied field discussed in Choe \& Lee (1996b). In a sheared magnetic arcade, the toroidal field $B_{z}$ is generally strongest near the polarity inversion line and decreases away from it. If resistivity is present, magnetic diffusion transports the toroidal flux outward and the poloidal field inward. The speeds of these transports depend on the spatial gradient of the toroidal field and the gradient of the flux function, i.e., the poloidal field strength. If the distance between adjacent field lines is large enough as in an elongated current layer, the transport of the toroidal flux is inefficient in the flux function $(\psi)$ space so that toroidal flux can be accumulated in a certain region in the $\psi$ space. This causes a further elongation of the current layer by dynamic evolution, which finally leads to initiation of magnetic reconnection. A footpoint convergence generally shrinks the whole system towards the origin and facilitates the transport of toroidal flux across field lines, which is unfavorable to reconnection trigger. Although both Case 2 and Case 3 involve converging motions, arcades in Case 2 already have a current layer before the initiation of the converging motion, but in Case 3 formation of a current layer is hindered by the footpoint convergence in the early stage of the evolution.

As the footpoints get closer to the origin, the converging speed is reduced and the shear growth is accelerated. Thus, less time is required for the trigger of the second arcade reconnection than the time required for the first reconnection trigger. However, the interval in units of $\bar{\zeta}_{m}$ between successive flaring events becomes larger with shift of reconnection events as shown in Figure 10, in which the height of O-lines is plotted versus $\bar{\zeta}_{m}$ the relative plasma displacement at the boundary normal field maxima. This is because the energy input by the converging motion is less effective in the presence of a magnetic island as discussed in $\S 5.1$. In Figure 10, the slopes of the curves both for integrated islands and for newborn islands tend to decrease with subsequent island generation, but one should not interpret this as decrease of the island rising speed. Since the relative plasma displacement is exponentially increasing with time due to the converging motion, the rising speed of the subsequently generated islands also increases. The rising speed of the newborn island in the fast rising phase is about $0.02 v_{0}$ in the first merging event and about $0.05 v_{0}$ in the second merging event. Readers are again reminded that the values of the speed can vary depending on the reconnection rate and the increasing trend is of most significance.

A direct comparison between results of different cases can not be simply made because the arcade system size varies case by case and also with time. From our findings, however, we can conclude that in the presence of a converging motion the individual flaring event tends to last 
longer and the energy release per event tends to be larger than in cases the magnetic shear is increased solely by a shearing motion.

\section{DISAPPEARANCE OF MAGNETIC ISLANDS BY DECREASE OF SHEAR: CASE 4}

It is also important to explore the evolution of an arcade system containing a magnetic island subject to a shear-decreasing footpoint motion. In this investigation, we first impose a shearing motion to a magnetic arcade with the same setting as employed in Case 1A in order to induce magnetic reconnection and create a magnetic island. The shearing motion is brought to an end at $t=28000 t_{0}$, which is a little while before the initiation of the second reconnection process in Case 1A. Then, a diverging footpoint motion is launched, which is given by equation (46) with $V_{x 0}=10^{-3} v_{0}$ and $x_{L}=18$. As shown in Figure 11, reconnection of line-tied field lines wrapping around the island continues and adds both poloidal and toroidal magnetic fluxes to the island system for a while after the commencement of the diverging motion although the magnetic shear, more exactly the toroidal flux, of the whole system is decreasing. This reconnection process originates from the overall expansion of the magnetic field. As shown in Low (1981) and Wu et al. (1986), the overall field configuration tends to scale up when the boundary flux distribution is extended. On the other hand, the field configuration tends to shrink down with reduction of the relative footpoint displacement. This tendency is strongest in the inner field lines that were vertically extended by magnetic shear. Just after the launch of the diverging motion, the expansion of the outer field lines causes the magnetic island to rise, which promotes the magnetic reconnection under the island. However, with a further reduction of the magnetic shear, the field lines under and near the magnetic island slowly fall down and a horizontally extended current layer develops between the magnetic island and the underlying arcade. Magnetic reconnection occurring in the current layer gradually dismantles the magnetic island. Finally, the magnetic island completely disappears as shown in Figure 11. The relative plasma displacement at the time of island disappearance is $\bar{\zeta}_{m} \sim 6$ in the shown case.

This result reminds us of an assertion by Aly (1990) that there exist more than one force-free field configuration with different field topologies if the footpoint shear of a magnetic arcade exceeds a certain critical value. These states are ideal MHD stable and a transition to another state is made by a flux-conserving magnetic reconnection. Although a proof to this assertion has not yet been given, a series of numerical experiments by Choe \& Lee (1996b) have provided a supporting evidence for it. This numerical study has found that whether magnetic reconnection can occur in an arcade to create a magnetic island does not depend on the resistivity value as long as it is nonzero, but on the amount of magnetic shear. A critical value of shear is also identified, below which magnetic reconnection is impossible regardless of the resistivity value. Of course, the critical value of shear must depend on the boundary flux distribution and the shear profile. Our numerical simulation adopts the same boundary flux distribution and shearing velocity profile as were employed by Choe 
\& Lee (1996b). The $\bar{\zeta}_{m}$ value for island disappearance in our study $\left(\bar{\zeta}_{m} \sim 6\right)$ is slightly less than the critical value $\left(\bar{\zeta}_{m} \sim 6.5\right)$ found by Choe \& Lee (1996b). However, this discrepancy is very natural because in our experiment, the magnetic shear in the magnetic island cannot be reduced by footpoint motions, but only through magnetic reconnection with line-tied fields. Thus, the value of relative plasm displacement for island dismantlement must be less than the value for the opposite process of island formation. Our simulation as well as the numerical experiment by Choe \& Lee (1996b) is not a perfect proof for Aly's assertion because a flux-conserving pointwise reconnection cannot be achieved in numerical simulations. However, since our numerical experiment is performed under a more relaxed condition than is assumed by Aly, our result gives a strong support for Aly's assertion.

\section{SUMMARY AND DISCUSSION}

In this paper, we have investigated the evolution of magnetic arcades under various shearincreasing and shear-reducing footpoint motions, focusing on dynamical interaction between magnetic islands and between a magnetic island and an ambient arcade. This study is particularly concerned with repeated flaring events in solar active regions, i.e., homologous flares. However, many physical processes revealed in our study are believed to be involved in general solar flares. Principal findings in our study are summarized as follows.

(1) When a magnetic arcade is subject to a continuing shear-increasing footpoint motion, generation of magnetic islands via magnetic reconnection and their coalescence can repeatedly take place with some time interval. The series of these reconnection processes is regarded to constitute a sequence of homologous flares.

(2) The rising of a newborn island comprises a slower first phase and a faster second phase. The first phase involves arcade field reconnection creating and adding flux to the new island and is identified with the preflare phase. Coalescence of two magnetic islands takes place within a short time span in the second phase, which is identified with the impulsive (or flash) phase of a flare. The island merging process creates a long and thin current sheet below and facilitates reconnection of the line-tied field. The obtained reconnection electric field is large enough to accelerate electrons to an energy level higher than $10 \mathrm{keV}$ required for observed X-ray emissions. The arcade field reconnection under the integrated island persists for quite a long time after the island merging, although the reconnection electric field gradually decays. This phase, in which more energy is released in total than in the earlier two phases, is identified with the main phase of a flare.

(3) The time interval between successive flaring events is shorter when the energy input rate into the system is higher. In the case that a pure shearing motion is imposed, more Poynting flux into the system is generated when reconnection of line-tied field is more active. Thus, the time interval between flaring events is in negative correlation with the magnetic reconnection rate.

(4) The energy input rate into the system depends on the type and pattern of the footpoint 
motion. Generally, a shearing motion produces more Poynting flux into the system than a converging motion when magnetic reconnection takes place in the presence of a magnetic island. Thus, individual flaring events have a longer duration and possibly release more energy when a converging motion contributes more to the shear-increasing process.

(5) When magnetic shear is decreased in a magnetic arcade containing a magnetic island, reconnection of the island field and the underlying line-tied field dismantles the magnetic island. This supports the idea that there is a critical amount of shear for existence of a magnetic island in an arcade-like field geometry.

It should be mentioned that the role of the upper magnetic island in our flare model can be performed by any magnetic flux system lying above the underlying arcade. The upper flux system may be connected to the interplanetary magnetic field or to distant magnetic poles on the solar surface. Thus, our flare model can explain the Yohkoh observation by Ohyama \& Shibata (1998) that the plasmoid motion comprises a slower first phase and a faster second phase. Such a two-step acceleration could not be interpreted tailored to the conventional picture of plasmoid formation such as Mikić \& Linker (1994), Choe \& Lee (1996b), and Amari et al. (1996a). Our numerical result indicates that the faster, second acceleration phase can be naturally achieved if the closed field lines above the plasmoid are reconnected with the further overlying flux. As discussed in $\S 4$, most flare energy in our simulation is released by the reconnection of line-tied field in a vertically elongated current sheet although the island coalescence is a more rapid process. Thus, our simulation results do not support the flux tube merging model of flares by Gold \& Hoyle (1960). However, the rapid rising of the magnetic island in a merging process plays a significant role in the fast reconnection in the impulsive (or flash) phase by stretching the arcade field lines so much as to form a thin and long current sheet. This is consistent with the plasmoid-induced-reconnection model of solar flares proposed by Shibata (1998). Our model, however, provides a plasmoid acceleration mechanism which was not given in Shibata's model.

In this paper, we have only considered situations, in which magnetic energy is supplied into the coronal magnetic field between the flaring events to recover the free energy released by the preceding flaring event. However, Moon et al. (1999) recently reported an observation that no indication of energy input, whether flux emergence or increase of magnetic shear, was detected throughout a series of flares. This may be attributed to the complex field geometry of the observed active region. In a complex active region containing more than a pair of magnetic poles, the transition to the lowest energy state may comprise several steps of macroscopic change in field topology. Thus, more observational studies are required to answer the question whether homologous flares are a repetitive process of energy input and release or a sequence of transitions towards the lowest energy state via several meta-stable states.

The rising of a magnetic island is a consequence of magnetic reconnection. However, one should not confuse the rising speed of the island with the reconnection outflow speed, which is the Alfvén speed upstream of the current sheet. Generally, the island moves much slower than 
the reconnection outflow. This is because the line-tied arcade field surrounding the island hinders the island from moving freely. The force causing the magnetic island movement is determined by the magnetic field enveloping the island (Choe \& Lee 1996b) and the island motion is a part of the global process approaching a new equilibrium. The dynamics of magnetic islands can be qualitatively understood by considering the currents in the system. The attraction of two islands before and during coalescence is quite natural because they have axial electric currents of the same direction. The rising of a single island, whether a newborn or an integrated one, can be understood with the concept of an imaginary current lying below the solar surface (Van Tend \& Kuperus 1978). The magnetic field generated by coronal currents cannot permeate the photosphere because of the high conductivity and large inertia in the solar interior. The fixed flux boundary condition in our simulation is actually the implementation of this high inductance condition in the photosphere. In this situation, the Lorentz force acting on a current carrying plasma can also be described as the force exerting on the coronal current by the image current of the opposite direction and the source current of the same direction generating the ambient potential field, both of which are located below the solar surface (Van Tend \& Kuperus 1978; Priest \& Forbes 1990; Forbes 1990). For the island rising motion to persist, the axial current flowing through the magnetic island must be increased. The increase of the axial current in the island can be ascribed to the increase of the magnetic helicity of the island. In our numerical study, helicity is assumed to be generated by footpoint motions. However, helicity can be directly injected into the corona by emergence of a pre-sheared field. Another possibility is that thin flux tubes emerge untwisted and not parallel to each other, i.e., having mutual helicities, and reconnect with each other to form a flux rope which has a sizable self-helicity (Song \& Lysak 1989; Chae 1999). These possibilities should be considered in future studies. With our results, however, we can at least assert that if homologous flares take place with a time interval far shorter than a day and no measurable shearing motion is observed in the photosphere, we have to consider helicity injection by emerging flux or self-helicity generation by reconnection of small scale flux tubes.

Although the magnetic island can move away from the sun as its current increases, it cannot go indefinitely far away unless the overlying arcade field lines open up. This is an important problem in relation to CMEs. Opening of the entire magnetic field is proved to be impossible (Aly 1991; Sturrock 1991), but the possibility of partial opening of the magnetic field has long been speculated (Low 1986; Wolfson \& Low 1992) and some numerical studies have produced supportive results in zero $\beta$ plasmas (Mikić \& Linker 1994; Roumeliotis, Sturrock, \& Antiochos 1994; Amari et al. 1996b). However, numerical computations in a finite domain, however large it may be, cannot tell whether the field opening is really a discontinuous process, i.e., a global singular nonequilibrium, or an asymptotic process. Aly (1995) discussed this problem thoroughly, but did not give a definite answer. Furthermore, no numerical studies have shown any tendency of field opening in a finite $\beta$ plasma (e.g., Mikić \& Linker 1994). How, then, can a plasmoid find its way out of the sun's magnetic barrier? In our simulation, we remark that the line-tied field surrounding the newborn island first reconnects with the field in the upper island before the merging of these two islands. Generally, coronal arcades are wrapped by open field lines which are believed to form a helmet 
streamer current sheet above the closed field structures. If this current sheet is an extended X-line and thus some wedge-like magnetic fields exist above this X-line, we can expect a rapid reconnection of the line-tied field below the X-line with the field above the X-line when a highly twisted helical magnetic structure is formed below. By this reconnection process, the field lines previously tied to the solar surface become open field lines. Then, the helical structure can freely move up either to reconnect with the remaining flux, if any, above the X-line or to navigate through the current sheet between the open field lines if no flux remains above the X-line. This scenario will be investigated in future studies. For the present, we expect that the result will be similar to what was found in the study on formation and acceleration of plasmoids in the earth's magnetotail by Otto, Schindler, \& Birn (1990). In their study, a plasmoid formed within an arcade-like tail escapes the siege of surrounding field lines owing to reconnection of these field lines with the field lines in the farther tail beyond the X-line. However, it needs to be confirmed by observation whether the helmet streamer current sheet really has an X-line-like structure and whether there exists magnetic flux above it.

This scenario can also be applied to a more probable case in which field lines surrounding the arcade are not open, but connected to magnetic poles located further outside. In this case, an $\mathrm{X}$-line exists above the arcade and an overlying arcade are present beyond the X-line. This type of field geometry was proposed in the solar eruption model of Antiochos, Devore, \& Klimchuk (1999), but they only considered the magnetic shear of closed fields in the underlying arcade. If a magnetic island (or a helical field loosely tied to the surface in 3D) is created in the underlying arcade, the rising of the island can induce reconnection of the underlying arcade field and the overlying arcade field. Then the field lines surrounding the magnetic island will be connected to magnetic poles far outside. To the magnetic island, this reconnection provides the same effect as field line opening in the sense that the closed magnetic barrier is removed. This possibility will also be investigated in future studies.

This work is supported by the DoE Contract No. DE-AC02-76-CHO3073 and the NSF grant ATM-9906142. 


\section{REFERENCES}

Aly, J. J. 1984, ApJ, 283, 349

-. 1990, Comput. Phys. Commun., 59, 13

—. 1991, ApJ, 375, L61

—. 1995, ApJ, 439, L63

Amari, T., Luciani, J. F., Aly, J. J., \& Tagger, M. 1996a, A\&A, 306, 913

-. 1996b, ApJ, 466, L39

Antiochos, S. K., Dahlburg, R. B., \& Klimchuk, J. A. 1994, ApJ, 420, L41

Antiochos, S. K., Devore, C. R., \& Klimchuk, J. A. 1999, ApJ, 485

Anzer, U. 1979, in Physics of Solar Prominences, IAU Colloquium 44, ed. E. Jensen, P. Maltby, \& F. Q. Orrall (Blindern-Oslo: Institute of Theoretical Astrophysics), 322

Biskamp, D. \& Welter, H. 1980, Phys. Rev. Lett., 44, 1069

—. 1989, Sol. Phys., 120, 49

Chae, J. 1999, in Physics of the Solar Corona and Transition Region Workshop, to be published

Cheng, C. Z. \& Choe, G. S. 1998, ApJ, 505, 376

Choe, G. S. \& Lee, L. C. 1992, Sol. Phys., 138, 291

-. 1996a, ApJ, 472, 360

-. 1996b, ApJ, 472, 372

Forbes, T. G. 1990, J. Geophys. Res., 95, 11919

Gold, T. \& Hoyle, F. 1960, MNRAS, 120, 89

Hagyard, M. J., Gaizauskas, V., Chapman, G. A., de Loach, A. C., Gary, G. A., Jones, H. P., Karpen, J. T., Martres, M.-J., Porter, J. G., Schmeider, B., Smith, Jr., J. B., \& Toomre, J. 1986, in Energetic Phenomena on the Sun, ed. M. Kundu \& B. E. Woodgate (Washington, DC: NASA CP-2439), 1

Hagyard, M. J., Teuber, D., West, E. A., \& Smith, J. B. 1984, Sol. Phys., 91, 115

Harned, D. S. \& Schnack, D. D. 1986, J. Comp. Phys., 65, 57

Inhester, B., Birn, J., \& Hesse, M. 1992, Sol. Phys., 138, 257 
Kan, J. R., Akasofu, S.-I., \& Lee, L. C. 1983, Sol. Phys., 84, 153

Kane, S. R. 1974, in Coronal Disturbances, IAU Symposium No. 57, ed. G. A. Newkirk (Dordrecht: D. Reidel), 105

Kopp, R. A. \& Pneuman, G. W. 1976, Sol. Phys., 50, 85

Kundu, M. \& Woodgate, B. E., eds. 1986, Energetic Phenomena on the Sun (Washington, DC: NASA CP-2439)

Kuperus, M. \& Raadu, M. A. 1974, A\&A, 315, 185

Lee, L. C., Choe, G. S., \& Akasofu, S.-I. 1995, in Space Plasmas: Coupling Between Small and Medium Scale Processes, ed. M. Ashour-Abdalla, T. Chang, \& P. Dusenbery (Washington, DC: American Geophysical Union), 29

Linker, J. A. \& Mikić, Z. 1995, ApJ, 438, L45

Livi, S. B., Martin, S., Wang, H., \& Ai, G. 1989, Sol. Phys., 121, 197

Lü, Y. P., Wang, J. X., \& Wang, H. N. 1993, Sol. Phys., 148, 119

Low, B. C. 1981, ApJ, 251, 352

—. 1986, ApJ, 307, 205

Martin, S. F. 1990, in Dynamics of Quiescent Prominences, IAU Colloquium 117, Lecture Notes in Physics, 363, ed. V. Ruždjak \& E. Tandberg-Hanssen (Berlin: Springer-Verlag), 1

Masuda, S., Kosugi, T., Hara, H., Tsuneta, S., \& Ogawara, Y. 1994, Nature, 371, 495

Mikić, Z., Barnes, D. C., \& Schnack, D. D. 1988, ApJ, 328, 830

Mikić, Z. \& Linker, J. A. 1994, ApJ, 430, 898

Moon, Y.-J., Yun, H. S., Choe, G. S., Park, Y. D., \& Mickey, D. L. 1999, Sol. Phys., submitted

Ohyama, M. \& Shibata, K. 1998, ApJ, 499, 934

Otto, A., Schindler, K., \& Birn, J. 1990, J. Geophys. Res., 95, 15023

Priest, E. R. 1982, Solar Magnetohydrodynamics (Dordrecht: D. Reidel)

Priest, E. R. \& Forbes, T. G. 1990, Sol. Phys., 126, 319

Roumeliotis, G., Sturrock, P. A., \& Antiochos, S. K. 1994, ApJ, 423, 847

Rust, D. M. \& Kumar, A. 1996, ApJ, 464, L199 
Shibata, K. 1998, in Observational Plasma Astrophysics: Five Years of Yohkoh and Beyond, ed. T. Watanabe, T. Kosugi, \& A. C. Sterling (Boston: Kluwer Academic Publishers), 187

Shibata, K., Masuda, S., Shimojo, M., Hara, H., Yokoyama, T., Tsuneta, S., Kosugi, T., \& Ogawara, Y. 1995, ApJ, 451, L83

Song, Y. \& Lysak, R. L. 1989, J. Geophys. Res., 94, 5273

Spicer, D. S. 1977, Sol. Phys., 53, 305

Stix, M. 1989, The Sun: An Introduction (Berlin: Springer-Verlag)

Sturrock, P. A. 1968, in Structure and Development of Solar Active Regions, IAU Symposium No. 35, ed. K. O. Kiepenheuer (Dordrecht: D. Reidel), 471

Sturrock, P. A. 1991, ApJ, 380, 655

Tsuneta, S. 1996, ApJ, 456, 840

Van Hoven, G. 1981, in Solar Flare Magnetohydrodynamics, ed. E. R. Priest (New York: Gordon and Breach), 217

Van Tend, W. \& Kuperus, M. 1978, Sol. Phys., 59, 115

Vekstein, G. E. \& Priest, E. R. 1992, ApJ, 384, 333

Wang, H. 1992, Sol. Phys., 140, 85

Wolfson, R. \& Low, B. C. 1992, ApJ, 391, 353

Woodgate, B. E. 1982, Adv. Space Res., 2, No. 11, 61

Woodgate, B. E., Martres, M.-J., Smith, Jr., J. B., Strong, K. T., McCabe, M. K., Machado, M. E., Gaizauskas, V., Stewart, R. T., \& Sturrock, P. A. 1984, Adv. Space Res., 4, No. 7, 11

Wu, S. T., Hu, Y. Q., Nakagawa, Y., \& Tandberg-Hanssen, E. 1986, ApJ, 306, 751

Zirin, H. 1988, Astrophysics of the Sun (Cambridge: Cambridge University Press) 
Table 1. Normalization Units

\begin{tabular}{lccc}
\hline \hline \multicolumn{1}{c}{ Quantity } & Symbol & Normalization Unit & Value \\
\hline Length & $\mathbf{x}$ & $L_{0}$ & $3.0 \times 10^{4} \mathrm{~km}$ \\
Magnetic Field & $\mathbf{B}$ & $B_{0}$ & $50 \mathrm{G}$ \\
Elec. No. Density & $n$ & $n_{0}$ & $1.0 \times 10^{9} \mathrm{~cm}^{-3}$ \\
Mass Density & $\rho$ & $\rho_{0}$ & $1.9 \times 10^{-15} \mathrm{~g} \mathrm{~cm}^{-3}$ \\
Pressure & $p$ & $p_{0}=B_{0}^{2} / 4 \pi$ & $2.0 \times 10^{2} \mathrm{dyne} \mathrm{cm}^{-2}$ \\
Temperature & $T$ & $T_{0}$ & $2.0 \times 10^{6} \mathrm{~K}^{-1}$ \\
Velocity & $\mathbf{v}$ & $v_{0}=B_{0} /\left(4 \pi \rho_{0}\right)^{1 / 2}$ & $3.2 \times 10^{3} \mathrm{~km} \mathrm{~s}^{-1}$ \\
Time & $t$ & $t_{0}=L_{0} / v_{0}$ & $9.3 \mathrm{~s}$ \\
Current Density & $\mathbf{J}$ & $J_{0}=(c / 4 \pi)\left(B_{0} / L_{0}\right)$ & $1.3 \times 10^{-4} \mathrm{~A} \mathrm{~m}^{-2}$ \\
Electric Field & $\mathbf{E}$ & $E_{0}=\left(v_{0} / c\right) B_{0}$ & $1.6 \times 10^{4} \mathrm{~V} \mathrm{~m}^{-1}$ \\
Energy & $W$ & $W_{0}=\left(B_{0}^{2} / 4 \pi\right) L_{0}^{3}$ & $5.4 \times 10^{30} \mathrm{erg}^{-1}$ \\
Poynting Vector & $\mathbf{S}$ & $S_{0}=\left(B_{0}^{2} / 4 \pi\right) v_{0}$ & $6.4 \times 10^{10} \mathrm{erg} \mathrm{cm}^{-2} \mathrm{~s}^{-1}$ \\
\hline
\end{tabular}


Table 2. Simulation Cases

\begin{tabular}{lccccccc}
\hline \hline Case & Motion $^{\mathrm{a}}$ & $a=\xi_{m}(t=0)^{\mathrm{b}}$ & $\eta^{\mathrm{c}}$ & $V_{z 0}{ }^{\mathrm{d}}$ & $V_{x 0}{ }^{\mathrm{e}}$ & $x_{L}{ }^{\mathrm{e}}$ & $\tau_{0}$ for $v_{x}{ }^{\mathrm{f}}$ \\
\hline $1 \mathrm{~A}$ & $\mathrm{~S}$ & 1 & $10^{-5}$ & $10^{-3}$ & $\mathrm{~N} / \mathrm{A}$ & $\mathrm{N} / \mathrm{A}$ & $\mathrm{N} / \mathrm{A}$ \\
$1 \mathrm{~B}$ & $\mathrm{~S}$ & 1 & $5 \times 10^{-5}$ & $10^{-3}$ & $\mathrm{~N} / \mathrm{A}$ & $\mathrm{N} / \mathrm{A}$ & $\mathrm{N} / \mathrm{A}$ \\
$1 \mathrm{C}$ & $\mathrm{S}$ & 1 & $10^{-5}$ & $5 \times 10^{-3}$ & $\mathrm{~N} / \mathrm{A}$ & $\mathrm{N} / \mathrm{A}$ & $\mathrm{N} / \mathrm{A}$ \\
$1 \mathrm{D}$ & $\mathrm{S}$ & 1 & 0 & $10^{-3}$ & $\mathrm{~N} / \mathrm{A}$ & $\mathrm{N} / \mathrm{A}$ & $\mathrm{N} / \mathrm{A}$ \\
$2 \mathrm{~A}$ & $\mathrm{C}$ after S & 3 & $10^{-5}$ & $10^{-3}$ & $10^{-3}$ & 18 & 28000 \\
$2 \mathrm{~B}$ & $\mathrm{C}$ after S & 3 & $5 \times 10^{-5}$ & $10^{-3}$ & $10^{-3}$ & 18 & 28000 \\
$2 \mathrm{C}$ & $\mathrm{C}$ after S & 3 & 0 & $10^{-3}$ & $10^{-3}$ & 18 & 28000 \\
3 & $\mathrm{C}$ and S & 3 & $10^{-5}$ & $10^{-3}$ & $5 \times 10^{-4}$ & 18 & 0 \\
4 & $\mathrm{D}$ after S & 1 & $10^{-5}$ & $10^{-3}$ & $10^{-3}$ & 18 & 28000 \\
\hline
\end{tabular}

${ }^{\text {a }} \mathrm{S}$ denotes a shearing motion, $\mathrm{C}$ denotes a converging motion and $\mathrm{D}$ denotes a diverging motion.

${ }^{\mathrm{b}}$ The initial (at $t=0$ ) maximum of the boundary normal field given by equation (34).

${ }^{c}$ Resistivity or the inverse of the Lundquist number.

dThe shearing speed at the boundary normal field maxima. See equation (43).

${ }^{\mathrm{e}} V_{x 0}=v_{x}\left(x=x_{L}, y=0\right)$. As seen in equation (46), the footpoint moving speed in the $x$-direction is proportional to $x$ for $|x| \leq x_{L}$.

${ }^{\mathrm{f}}$ The commencing time for $v_{x}$ at the bottom boundary. 


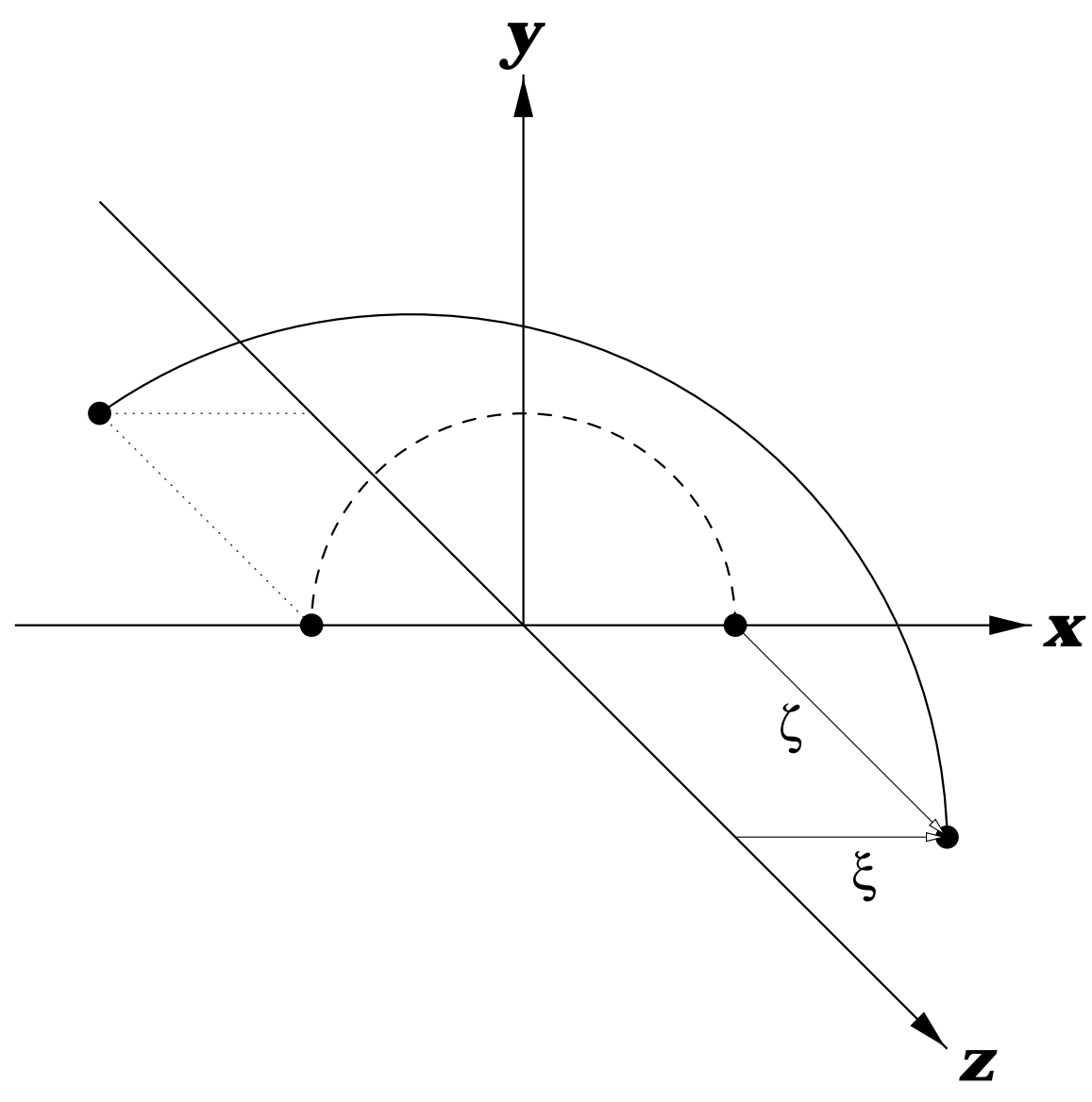

Fig. 1. - A sketch of a field line in our coordinate system. The solar surface is modeled as the $y=0$-plane, in which the polarity inversion line is along the $z$-axis. The field line of the initial potential field is represented by a dashed line and the field line at a later time $t>0$ by a solid line. The distance, over which a fluid element on the solar surface has moved in the $z$-direction is denoted by $\zeta$, which is equal to half the distance in the $z$-direction between two conjugate footpoints as shown in the figure under the ideal MHD condition. The distance between a field line footpoint and the polarity inversion line is denoted by $\xi$. 


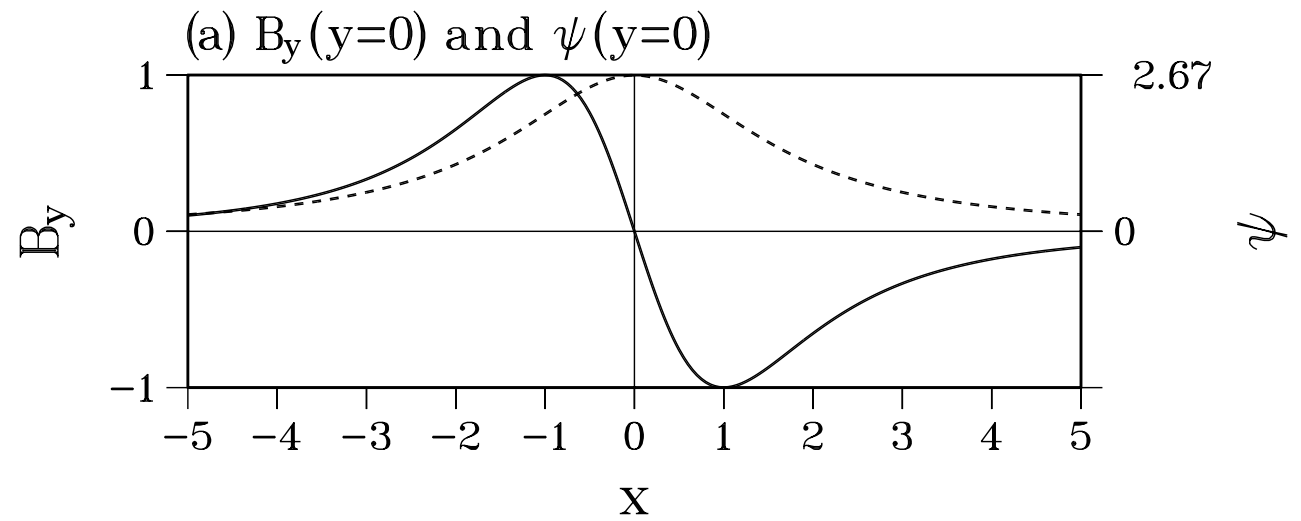

(b) $V_{z}(y=0)$
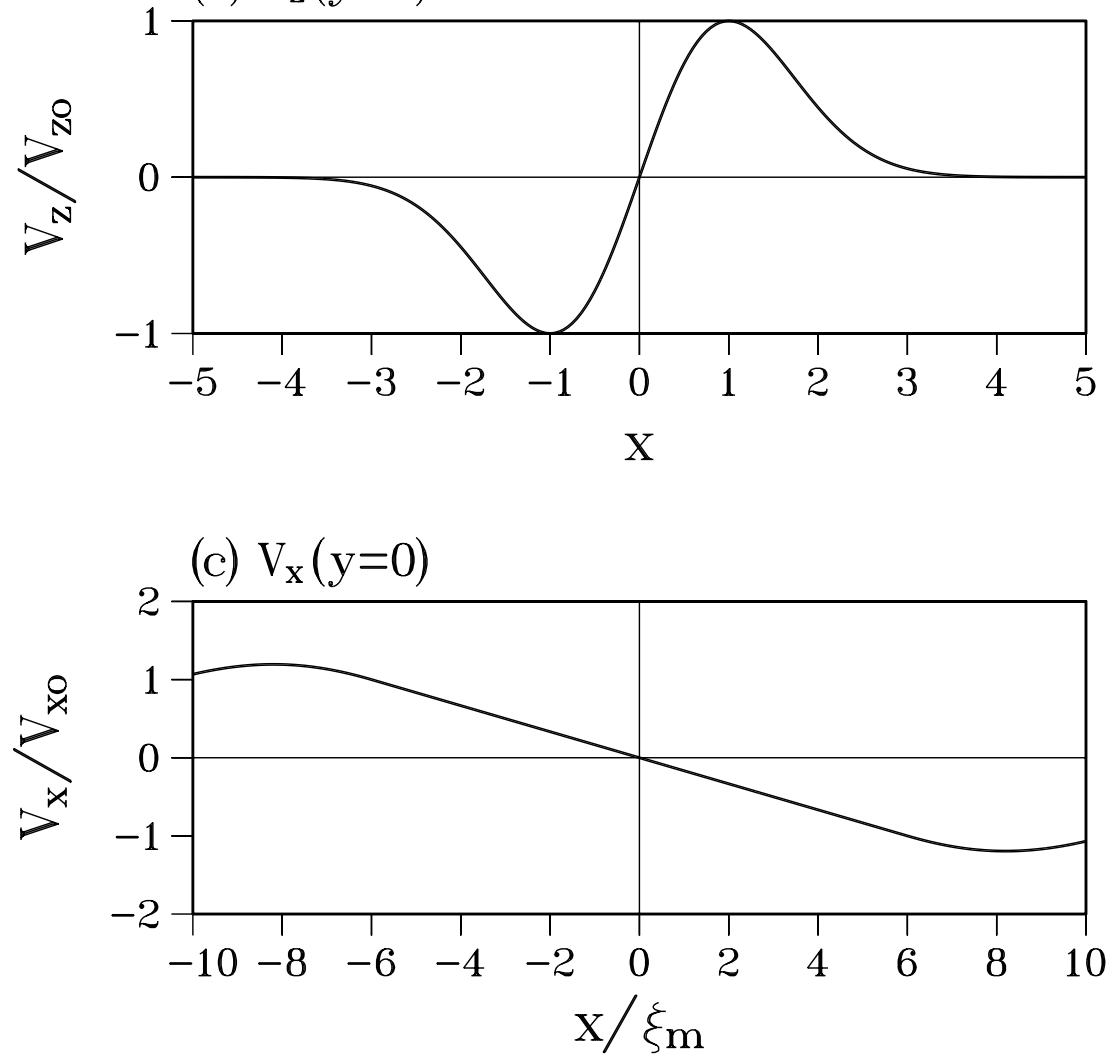

Fig. 2.- Profiles of (a) boundary normal magnetic field (solid line) and flux through the bottom boundary (dashed line), (b) shearing velocity in the $z$-direction, and (c) converging (or diverging) velocity in the $x$-direction. The normalization of all the quantities is given in table 1. 

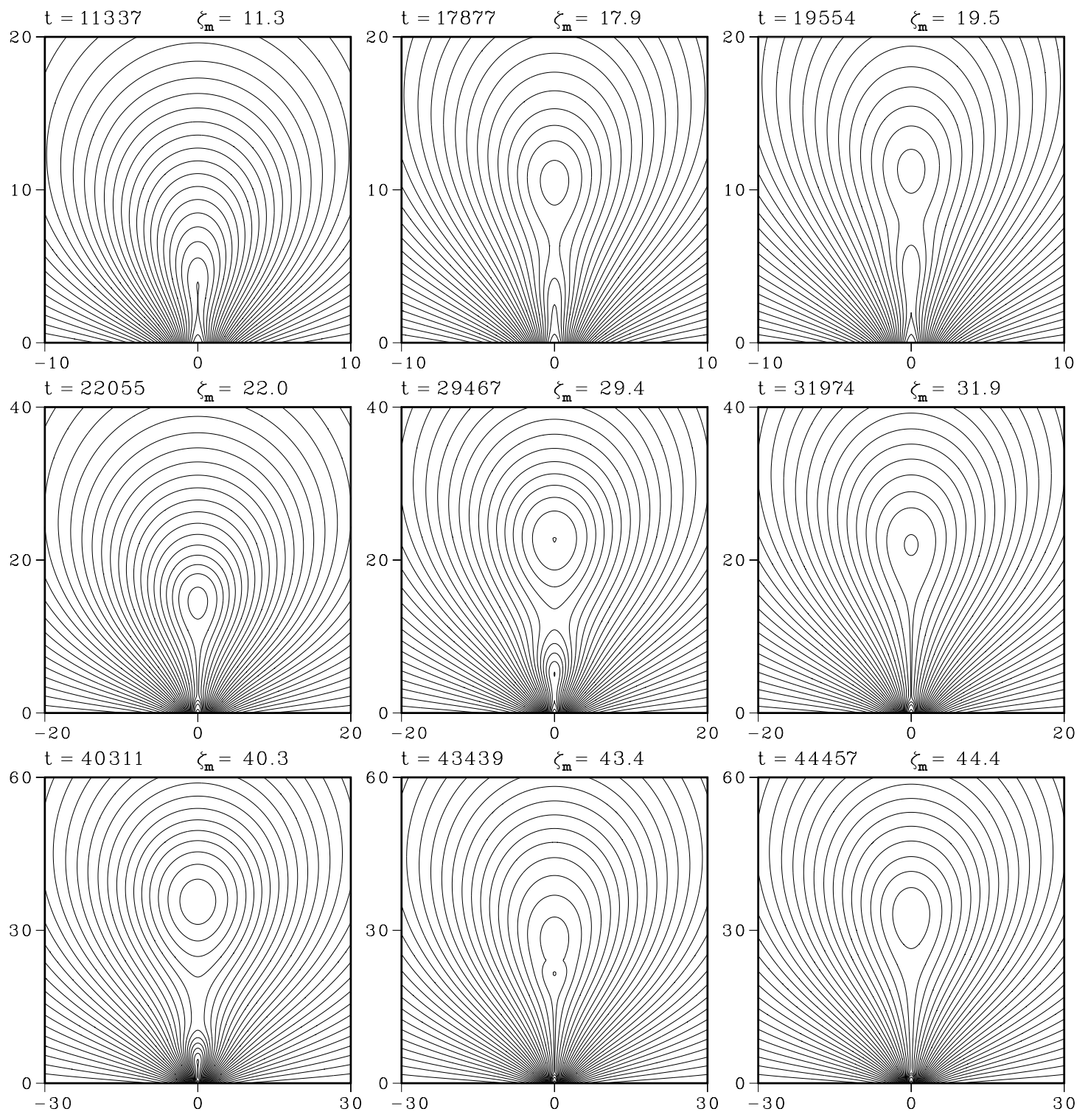

Fig. 3.- Repeated formation of magnetic islands and their merging under continuous footpoint shearing for Case 1A. Field lines projected on the $x y$-plane are shown for different times. Note that the figure scale increases row by row. The plasma displacement in the $z$-direction at the boundary normal field maxima $(x= \pm 1)$ is denoted by $\zeta_{m}$. 


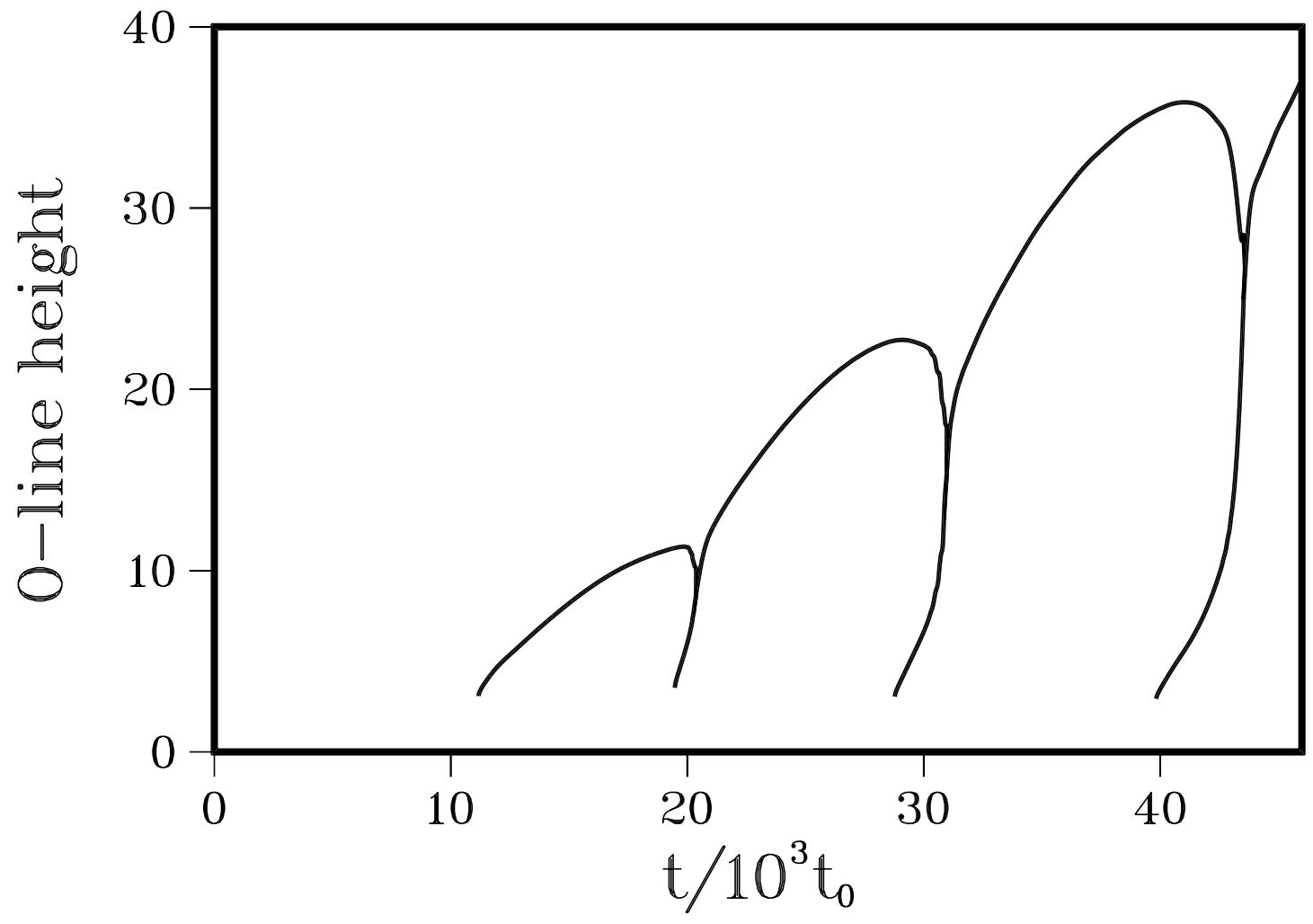

Fig. 4. - The height of O-lines of the magnetic islands in Case 1A as a function of time. The slope of the curves represents the rising speed of the magnetic islands. 

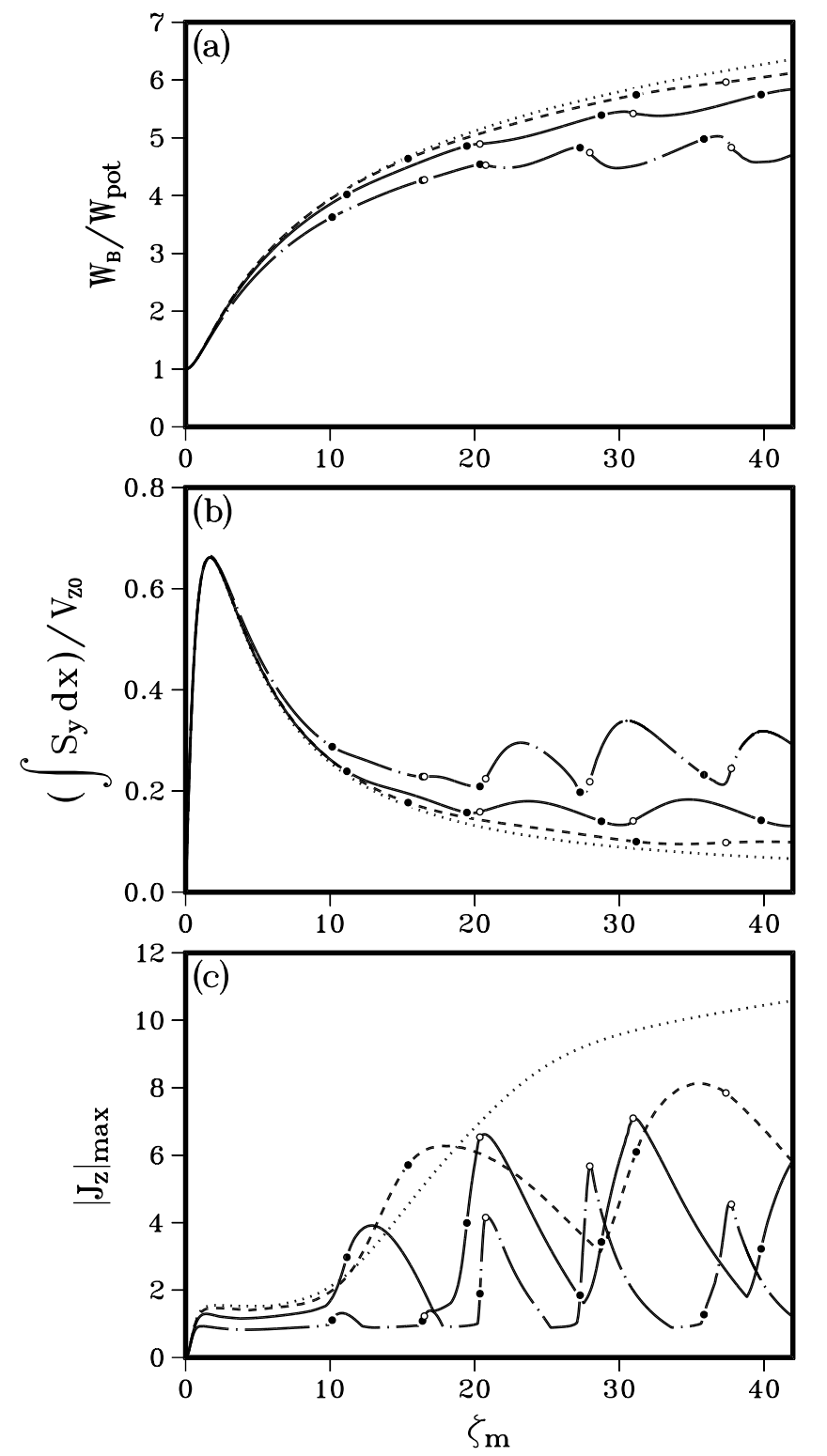

Fig. 5. - The evolution of (a) magnetic energy in units of the potential field energy, (b) Poynting flux through the bottom boundary divided by $V_{z 0}$, and (c) maximum magnitude of the current density in the current sheet under the magnetic island for Case 1's. All the quantities are plotted as a function of $\zeta_{m}$ which is the plasma displacement at $x= \pm 1$. In all three figures, the solid lines represent Case $1 \mathrm{~A}$ in which $V_{z 0}=10^{-3} v_{0}$ and $\eta=10^{-5}$, the chain-dotted lines represent Case $1 \mathrm{~B}$ in which $V_{z 0}=10^{-3} v_{0}$ and $\eta=5 \times 10^{-5}$, and the dashed lines represent for Case $1 \mathrm{C}$ in which $V_{z 0}=5 \times 10^{-3} v_{0}$ and $\eta=10^{-5}$. The dotted lines are for the ideal MHD case (Case 1D) in which $V_{z 0}=10^{-3} v_{0}$ and $\eta=0$. The filled circles denote the initiation of a new reconnection event in the underlying arcade and the blank circles indicate the completion of the island merging. 

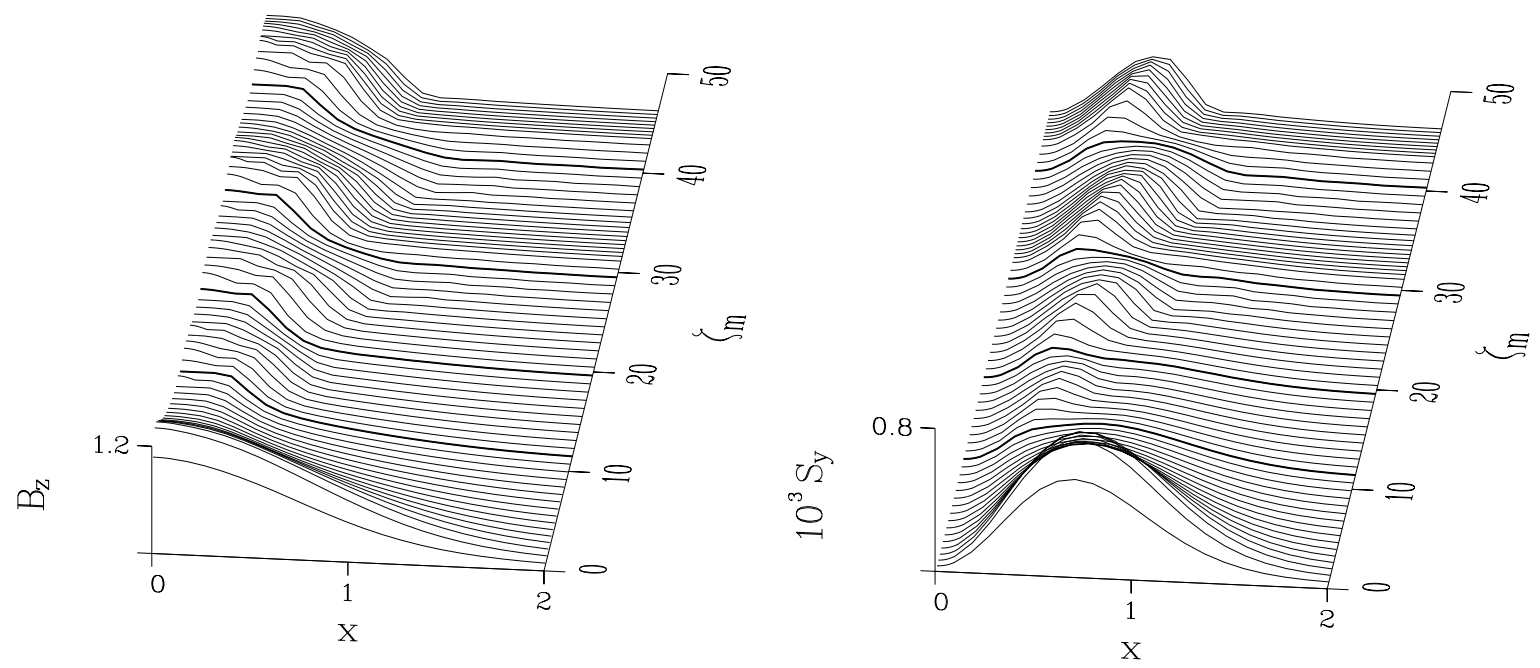

Fig. 6. - The toroidal magnetic field $\left(B_{z}\right)$ and the upward component of the Poynting vector $\left(S_{y}\right)$ in Case $1 \mathrm{~A}$ plotted along the bottom boundary $(0 \leq x \leq 2, y=0)$ for different plasma displacements $\left(\zeta_{m}\right)$, which is almost proportional to time. Thick lines indicate the initiation of a new reconnection in the underlying arcade. 

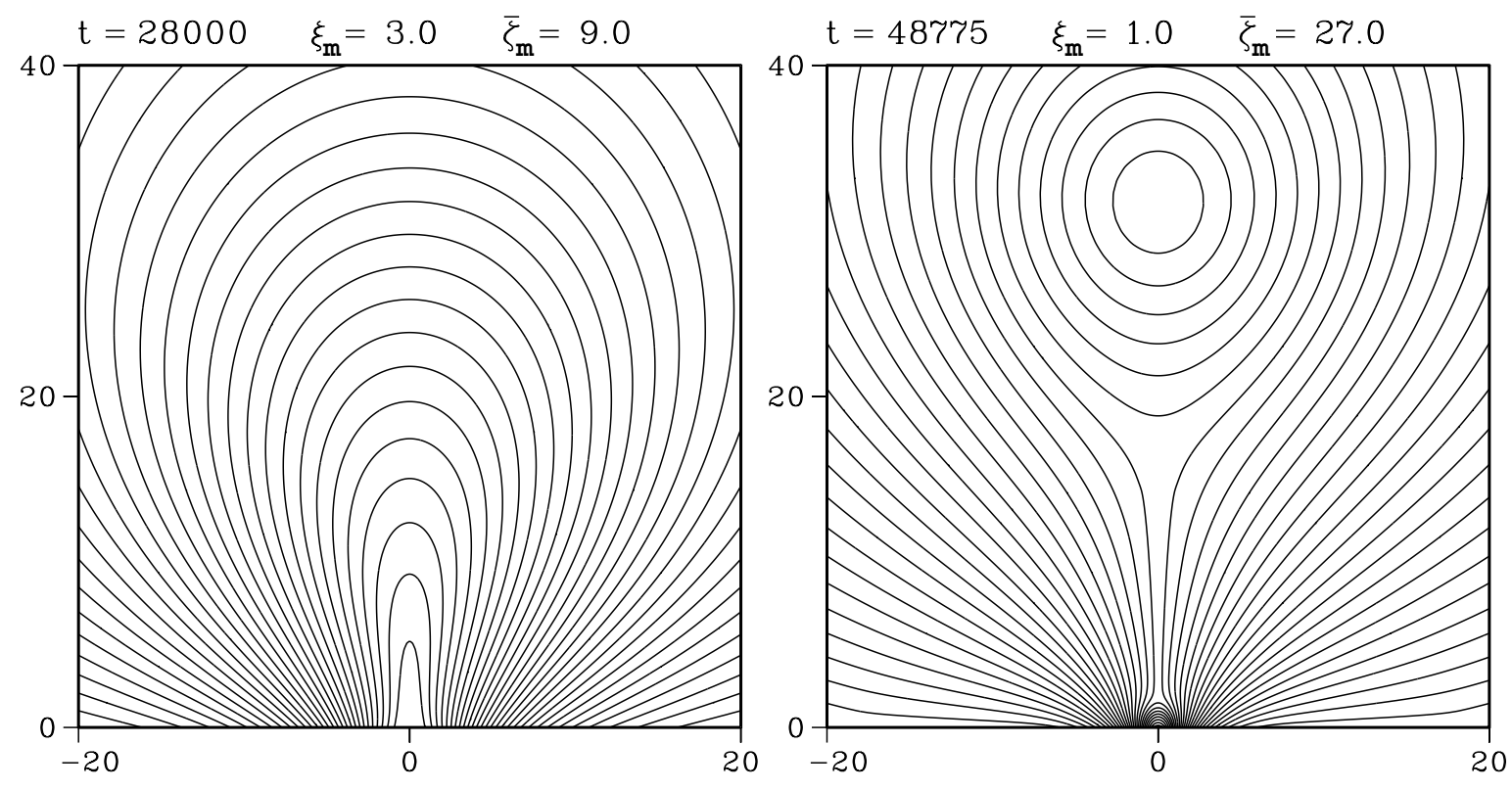

Fig. 7.- The resistive evolution of a magnetic arcade under a converging footpoint motion for $\eta=10^{-5}$ (Case 2A). In this case, a shearing footpoint motion parallel to the polarity inversion line with $V_{z 0}=10^{-3} v_{0}$ is first imposed for $\bar{\zeta}_{m}=\zeta_{m} / \xi_{m}<9$. Then, a purely converging footpoint motion given by equation (46) is applied to the arcade to further increase the relative plasma displacement. 


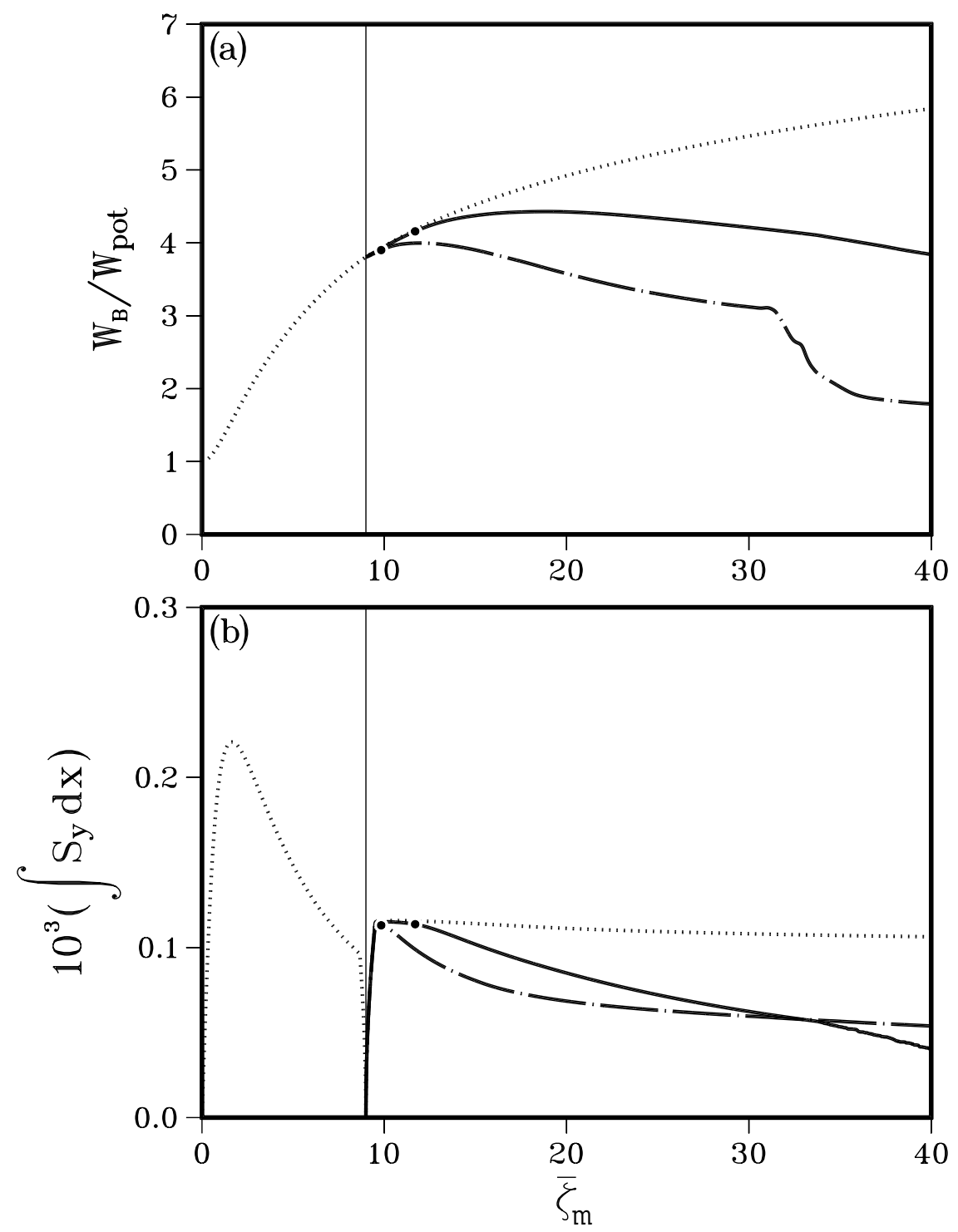

Fig. 8.- (a) The magnetic energy in units of the potential field energy and (b) the Poynting flux through the bottom boundary as a function of $\bar{\zeta}_{m}$ for Case 2's. In both figures, the solid lines represent Case 2A in which $\eta=10^{-5}$ and the chain-dotted lines represent Case $2 \mathrm{~B}$ in which $\eta=5 \times 10^{-5}$. The dotted lines represent the ideal MHD case (Case $2 \mathrm{C}$ ). In all three cases, the same boundary velocity profile is imposed. The vertical line indicates the time at which the shearing motion ceases and the converging motion commences. The initiation of magnetic reconnection is marked with a filled circle. 

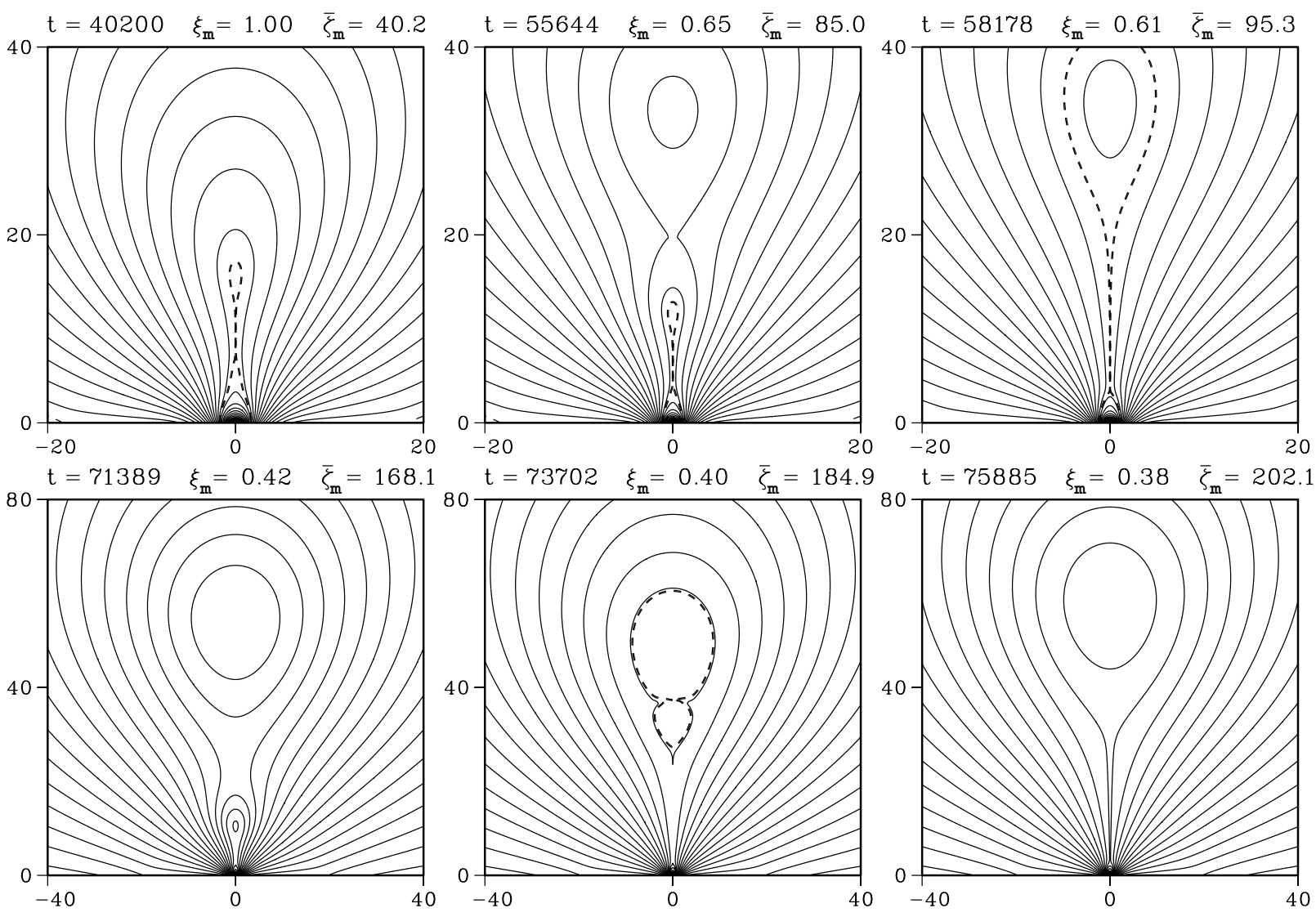

Fig. 9.- Repetitive formation and merging of magnetic islands in a magnetic arcade subject to simultaneous shearing and converging footpoint motions with resistivity $\eta=10^{-5}$ (Case 3 ). In this case, the shearing velocity of each field line footpoint is maintained as given by eq. (43) while the footpoint undergoes a converging motion. The maximum shearing speed is $V_{z 0}=10^{-3} v_{0}$ at the $B_{y}$ maxima and the converging speed increases linearly with $|x|$ up to $V_{x 0}=5 \times 10^{-4} v_{0}$ at $x= \pm 18$. Field lines are drawn with a constant increment in poloidal flux value, but when necessary, extra field lines are drawn in dashed lines in order to show a more detailed field geometry. 


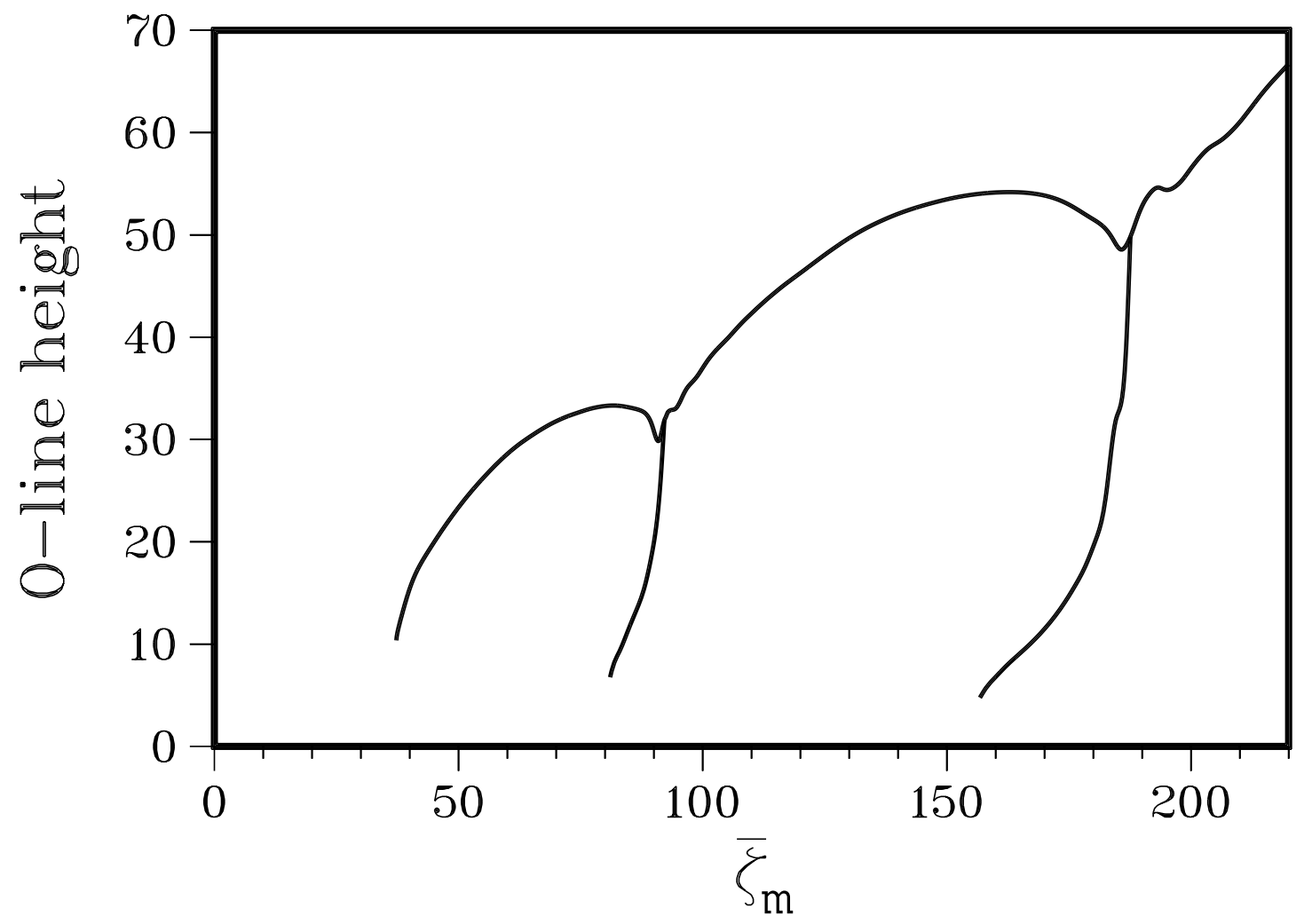

Fig. 10. - The height of O-lines in the magnetic islands in Case 3 as a function of $\bar{\zeta}_{m}$ the relative plasma displacement at the boundary normal field maxima. Since $\bar{\zeta}_{m}$ increases almost exponentially with time in this case, the rising speed of the magnetic islands increases more with shift in their generation than in Case 1. 

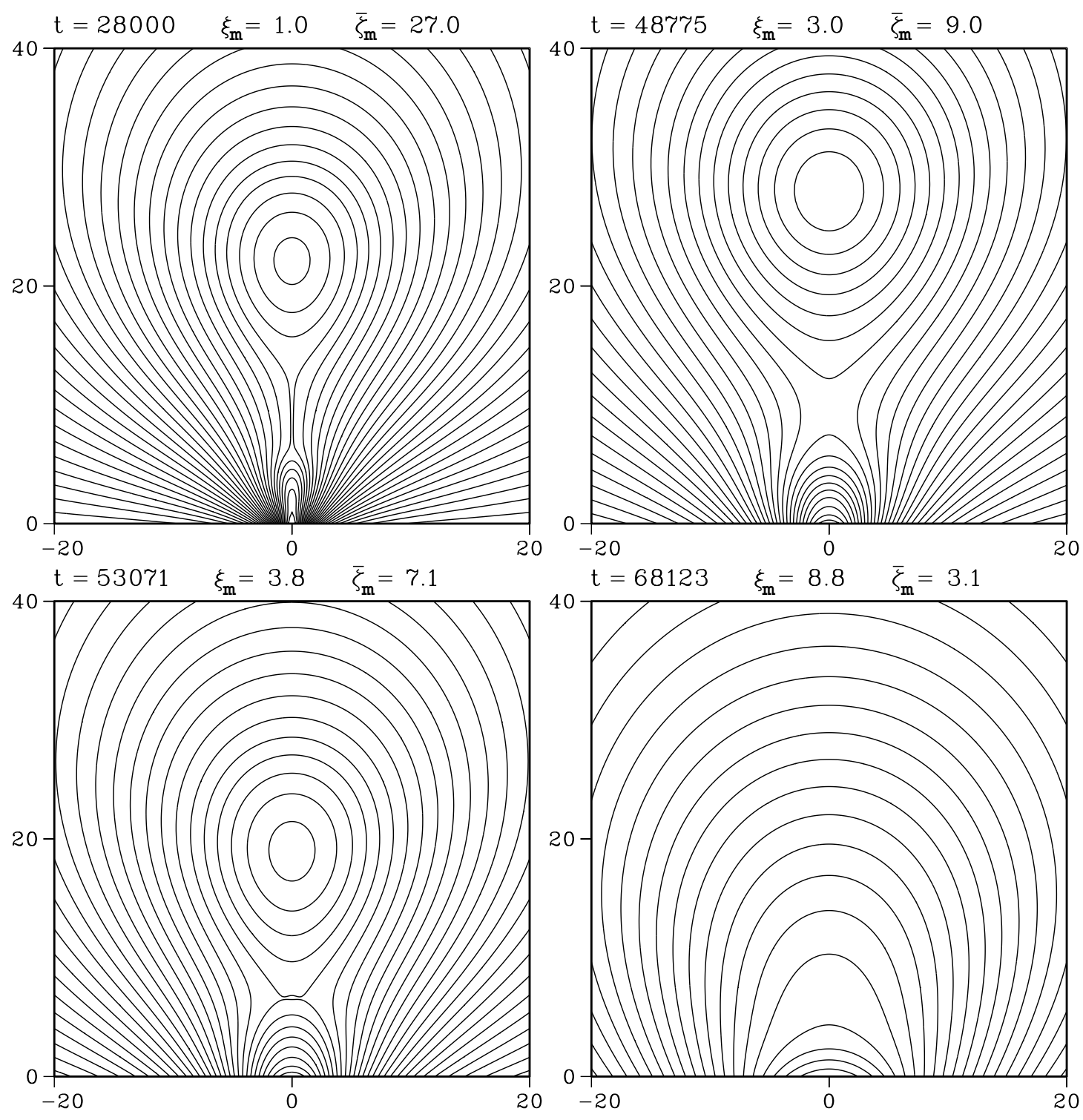

Fig. 11.- Destruction of the magnetic island in a magnetic arcade undergoing a shear-reducing, diverging footpoint motion (Case 4). In this case, we first impose a shearing footpoint motion for $\eta=10^{-5}$ to create a magnetic island. Then, we turn off the shearing motion and impose a diverging footpoint motion with $V_{x 0}=10^{-3} v_{0}$ at $x= \pm 18$ to reduce the relative plasma displacement. The magnetic island disappears at $\bar{\zeta}_{m} \sim 6$. 